\title{
DIGITALCOMMONS
}

@WAYNESTATE —

Wayne State University

$2-1-2010$

\section{Enhanced Metric Regularity and Lipschitzian Properties of Variational Systems}

Francisco J. Aragón Artacho

University of Alicante, Spain, francisco.aragon@ua.es

Boris S. Mordukhovich

Wayne State University, boris@math.wayne.edu

\section{Recommended Citation}

Aragón Artacho, Francisco J. and Mordukhovich, Boris S., "Enhanced Metric Regularity and Lipschitzian Properties of Variational Systems" (2010). Mathematics Research Reports. Paper 73.

http://digitalcommons.wayne.edu/math_reports/73

This Technical Report is brought to you for free and open access by the Mathematics at DigitalCommons@WayneState. It has been accepted for inclusion in Mathematics Research Reports by an authorized administrator of DigitalCommons@WayneState. 


\section{ENHANCED METRIC REGULARITY AND LIPSCHITZIAN PROPERTIES OF VARIATIONAL SYSTEMS}

FRANCISCO J. ARAGÓN ARTACHO and BORIS S. MORDUKHOVICH

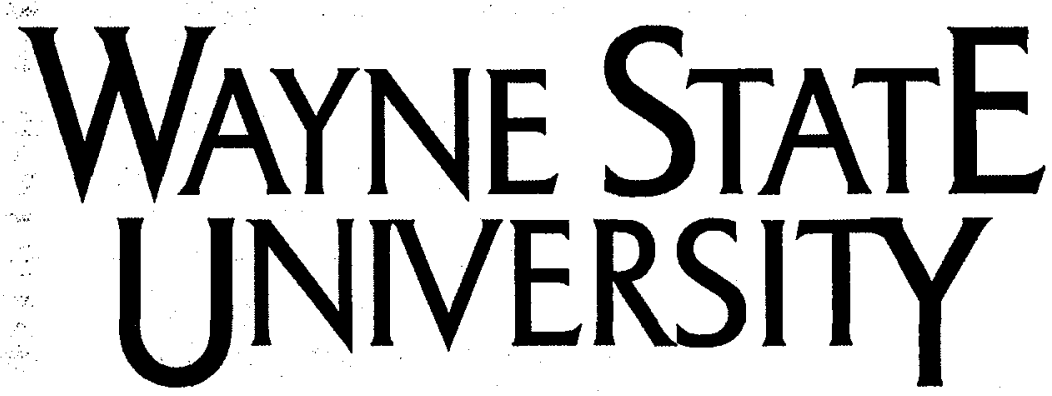

Detroit, MI 48202

Department of Mathematics

Research Report

\section{Series}

\#2

This research was partly supported by the USA National Science Foundation 


\title{
ENHANCED METRIC REGULARITY AND LIPSCHITZIAN PROPERTIES OF VARIATIONAL SYSTEMS
}

\author{
FRANCISCO J. ARAGÓN ARTACHO
}

Department of Statistics and Operations Research, University of Alicante, 03071 Alicante, Spain

Email: francisco.aragon@ua.es

and

\section{BORIS S. MORDUKHOVICH}

Department of Mathematics, Wayne State University, Detroit, MI 48202, USA

Email: boris@math.wayne.edu

\begin{abstract}
This paper mainly concerns the study of a large class of variational systems governed by parametric generalized equations, which encompass variational and hemivariational inequalities, complementarity problems, first-order necessary optimality conditions, and other optimizationrelated models important for optimization theory and applications. An efficient approach to these issues has been developed in our preceding work [1] establishing qualitative and quantitative relationships between conventional metric regularity/subregularity and Lipschitzian/calmness properties in the framework of parametric generalized equations in arbitrary Banach spaces. This paper provides, on one hand, significant extensions of the major results in [1] to new partial metric regularity and hemiregularity properties. On the other hand, we establish enhanced relationships between certain strong counterparts of metric regularity/hemiregularity and single-valued Lipschitzian localizations. The results obtained are new in both finite-dimensional and infinite-dimensional settings.
\end{abstract}

Keywords. Variational analysis and optimization, parametric variational systems, generalized equations, set-valued mappings, metric regularity, Lipschitzian properties 


\section{Introduction}

In this paper we study a broad class of parametric variational systems defined by

$$
0 \in f(x, y)+Q(y)
$$

where $Y \in Y$ is a decision variable, $x \in X$ is a parameter, $f: X \times Y \rightarrow Z$ is a single-valued "base" mapping, and $Q: Y \rightrightarrows Z$ is a set-valued "field" mapping between arbitrary Banach spaces. Models of this type have been introduced and studied by Robinson in the late 1970s (see [6] and its references) under the name of "generalized equations." Since that, they have been extensively developed and applied to numerous issues of variational analysis, optimization, equilibria, etc.; see, e.g., the books $[2,3,4]$ and the bibliographies therein.

It has been well recognized that the generalized equation model (1.1) is a common and convenient framework for studying particular classes of parametric variational systems. We mention variational inequalities corresponding to the normal cone mapping $Q(y)=N(y ; \Omega)$ to a convex set $\Omega$ in (1.1), hemivariational inequalities with $Q(y)=\partial \varphi(y)$ defined by a subdifferential of some function $\varphi$, complementarity problems with $\Omega=\mathbb{R}_{+}^{n}$ in the above normal cone description, $K K T$ systems (first-order optimality conditions) in parametric nonlinear programming, etc.

Associated with (1.1), define the parameter-dependent solution map $S: X \rightrightarrows Y$ by

$$
S(x):=\{y \in Y \mid 0 \in f(x, y)+Q(y)\} .
$$

In [1], we established various qualitative and quantitative relationships between fundamental metric regularity properties of the solution maps (1.2) and Lipschitzian properties of the field mappings $Q$ of the generalized equations (1.1), and vice versa.

This paper continues our study in two major directions. On one hand, we extend some important results of [1] to new notions of partial metric regularity and hemiregularity of the solution and field mappings in (1.1) and illuminate their connections to the corresponding Lipschitzian/calmness behavior. On the other hand, we consider certain strong counterparts of the aforementioned metric regularity/hemiregularity properties, establishing their qualitative and quantitative relationships with single-valued Lipschitzian/calmness localizations. Our approach to these issues is based on developing appropriate versions of the Lyusternik-Graves iterative process in general Banach spaces.

The rest of the paper is organized as follows. Section 2 contains some preliminary material, mostly based on [1], needed in what follows. In Section 3 we introduce the notion of partial regularity for set-valued mappings and use it to extend some major results of [1]. Section 4 is devoted to the study and applications of the notions of strong metric regularity and strong metric subregularity and their qualitative and quantitative relationships with single-valued Lipschitzian localizations in the framework of the parametric variational systems (1.1). The final Section 5 concerns new notions of metric hemiregularity and strong metric hemiregularity and the corresponding Lipschitzian/calmness properties in the variational setting of (1.1).

Our notation is basically standard in variational analysis, expect new symbols defined in the appropriate places. Recall that $\mathbb{B}_{\alpha}(\bar{x})$ and $\mathbb{B}$ stand, respectively, for the closed unit ball and the closed ball centered at $\bar{x}$ with radius $\alpha>0$ in the space in question, that $\mathbb{R}:=\mathbb{R} \cup\{\infty\}$ denotes the extended real line, that $\mathcal{L}(X, Y)$ stands for the collection of linear bounded operators $A: X \rightarrow Y$ between Banach spaces, and that $\mathbb{N}:=\{1,2, \ldots\}$ is the set of natural numbers. 


\section{$2 \quad$ Background material}

Let us first recall some notions used in what follows. We refer the reader to $[1,2,4]$ for more details, discussions, and references regarding these and related notions of variational analysis.

A set-valued mapping $F: X \rightrightarrows Y$ between Banach spaces is said to be metrically regular around a point $(\bar{x}, \bar{y}) \in \operatorname{gph} F$ from its graph

$$
\operatorname{gph} F:=\{(x, y) \in X \times Y \mid y \in F(x)\}
$$

with constant $\kappa>0$ if there are neighborhoods $U \subset X$ of $\bar{x}$ and $V \subset Y$ of $\bar{y}$ such that

$$
d\left(x, F^{-1}(y)\right) \leq \kappa d(y, F(x)) \text { for all } x \in U \text { and } y \in V,
$$

where $d(\cdot ; \Omega)$ stands for the distance function associated with a set $\Omega$. The infimum of $\kappa>0$ over all the combinations $(\kappa, U, V)$ for which $(2.1)$ holds is called the EXACT REGULARITY BOUND of $F$ around $(\bar{x}, \bar{y})$ and is denoted $\operatorname{reg} F(\bar{x}, \bar{y})$.

We say that $F$ is metrically regular at $(\bar{x}, \bar{y}) \in \operatorname{gph} F$ (or metrically subregular at this point) with constant $\kappa>0$ if there is a neighborhood $U$ of $\bar{x}$ such that

$$
d\left(x, F^{-1}(\bar{y})\right) \leq \kappa d(\bar{y}, F(x)) \text { for all } x \in U .
$$

The infimum of $\kappa>0$ over all the combinations $(\kappa, U)$ for which (2.2) holds is called the EXACT SUBREGULARITY BOUND of $F$ at $(\bar{x}, \bar{y})$ and is denoted subreg $F(\bar{x}, \bar{y})$.

Recall further that a single-valued mapping $f: X \times Y \rightarrow Z$ is (partially) Lipschitz continuous around $(\bar{x}, \bar{y})$ with respect to $x$ uniformly in $y$ if there are neighborhoods $U$ of $\bar{x}$ and $V$ of $\bar{y}$ along with a constant $\eta \geq 0$ such that

$$
\left\|f(x, y)-f\left(x^{\prime}, y\right)\right\| \leq \eta\left\|x-x^{\prime}\right\| \text { whenever } x, x^{\prime} \in U \text { and } y \in V .
$$

The infimum of $\eta$ over all such combinations of $\eta, U$, and $V$ in (2.3) is called the (exact) partial uniform Lipschitz modulus of $f$ in $x$ around $(\bar{x}, \bar{y})$ and is denoted $\widehat{\operatorname{lip}}_{x} f(\bar{x}, \bar{y})$. The corresponding Lipschitz property of $f$ with respect to $y$ and the modulus $\widehat{\operatorname{lip}}_{y} f(\bar{x}, \bar{y})$ are defined similarly.

A set-valued mapping $F: X \rightarrow Y$ is Lipschitz-like around $(\bar{x}, \bar{y}) \in \operatorname{gph} F$ (or it has the Aubin property around this point) constant $\ell \geq 0$ if there are neighborhoods $U$ of $\bar{x}$ and $V$ of $\bar{y}$ such that

$$
F(x) \cap V \subset F\left(x^{\prime}\right)+\ell\left\|x-x^{\prime}\right\| \mathbb{B} \text { for all } x, x^{\prime} \in U .
$$

The infimum of $\ell \geq 0$ over all the combinations $(\ell, U, V)$ for which (2.4) holds is called the EXACT LiPSCHITZIAN BOUND of $F$ around $(\bar{x}, \bar{y})$ and is denoted $\operatorname{lip} F(\bar{x}, \bar{y})$. Similarly to $(2.3)$ we define the partial Lipschitz-like property of $F: X \times Y \Rightarrow Z$ and its exact bound.

It is said that $F$ is calm at $(\bar{x}, \bar{y}) \in \operatorname{gph} F$ with constant $\ell \geq 0$ if there are neighborhoods $U$ of $\bar{x}$ and $V$ of $\bar{y}$ such that

$$
F(x) \cap V \subset F(\bar{x})+\ell\|x-\bar{x}\| \mathbb{B} \text { for all } x \in U .
$$

The infimum of $\ell \geq 0$ over all the combinations $(\ell, U, V)$ for which (2.5) holds is called the EXACT BOUND OF CALMNESS for $F$ at $(\bar{x}, \bar{y})$ and is denoted $\operatorname{clm} F(\bar{x}, \bar{y})$.

Similarly to (2.3) we define the corresponding versions of the partial calmness properties of $f: X \rightarrow Y$ with moduli $\widehat{\operatorname{clm}}_{x} f(\bar{x}, \bar{y})$ and $\widehat{\operatorname{clm}}_{y} f(\bar{x}, \bar{y})$, respectively.

The following result was obtained in [1, Lemma 3.1 and Remark 3.2] by using a certain modification of the Lyusternik-Graves iterative process. 
Theorem 2.1 (implicit multifunctions). Let $f: X \times Y \rightarrow Z$ be a mapping between Banach spaces, and let $(\bar{x}, \bar{y}) \in X \times Y$ be such that $f$ is locally Lipschitzian with respect to $y$ with constant $\eta \geq 0$ uniformly in $x$ on some neighborhood $U \times V$ of $(\bar{x}, \bar{y})$. Given a surjective linear operator $A \in \mathcal{L}(X, Z)$, suppose that there are $\mu \geq 0$ and $\gamma>\operatorname{reg} A$ satisfying the relationships $\mu \gamma<1$ and

$$
\left\|f(x, y)-f\left(x^{\prime}, y\right)-A\left(x-x^{\prime}\right)\right\| \leq \mu\left\|x-x^{\prime}\right\| \text { for all } x, x^{\prime} \in U \text { and } y \in V .
$$

Given further a mapping $g: W \rightarrow Z$ between Banach spaces that is locally Lipschitzian around $\bar{w} \in W$ with constant $\lambda$, consider a set-valued mapping $\Gamma: Y \times W \rightrightarrows X$ defined by

$$
\Gamma(y, w):=\{x \in X \mid f(x, y)+g(w)=0\} .
$$

Then there is $\alpha>0$ such that for every $(y, w),\left(y^{\prime}, w^{\prime}\right) \in \mathbb{B}_{\alpha}(\bar{y}) \times \mathbb{B}_{\alpha}(\bar{w})$ we have the inclusion

$$
\Gamma\left(y^{\prime}, w^{\prime}\right) \cap \mathbb{B}_{\alpha}(\bar{x}) \subset \Gamma(y, w)+\frac{\gamma}{1-\gamma \mu}\left(\eta\left\|y-y^{\prime}\right\|+\lambda\left\|w-w^{\prime}\right\|\right) \mathbb{B}
$$

The latter implies, when $g(\bar{w})=-f(\bar{x}, \bar{y})$, that $\Gamma$ is Lipschitz-like around $((\bar{y}, \bar{w}), \bar{x})$ with the following upper estimate of the exact Lipschitzian bound:

$$
\operatorname{lip} \Gamma((\bar{y}, \bar{w}), \bar{x}) \leq \frac{\operatorname{reg} A \cdot \max \left\{\widehat{\operatorname{lip}}_{y} f(\bar{x}, \bar{y}), \operatorname{lip} g(\bar{w})\right\}}{1-\mu \cdot \operatorname{reg} A} .
$$

Furthermore, under the weaker assumptions

$$
\widehat{\operatorname{clm}}_{y} f(\bar{x}, \bar{y})<\eta \text { and } \operatorname{clm} g(\bar{w})<\lambda
$$

we have the weaker counterpart of inclusion (2.8): there is $\alpha>0$ such that

$$
\Gamma(y, w) \cap \mathbb{B}_{\alpha}(\bar{x}) \subset \Gamma(\bar{y}, \bar{w})+\frac{\gamma}{1-\gamma \mu}(\eta\|y-\bar{y}\|+\lambda\|w-\bar{w}\|) \mathbb{B}
$$

for every $(y, w) \in \mathbb{B}_{\alpha}(\bar{y}) \times \mathbb{B}_{\alpha}(\bar{w})$.

It can be easily observed from the proof [1] of Theorem 2.1 that removing the Lipschitz assumption on $f$ therein, we get instead (2.8) the following conclusion: there is $\alpha>0$ such that

$$
\Gamma\left(y, w^{\prime}\right) \cap \mathbb{B}_{\alpha}(\bar{x}) \subset \Gamma(y, w)+\frac{\gamma}{1-\gamma \mu} \lambda\left\|w-w^{\prime}\right\| \mathbb{B} \text { for all } y \in \mathbb{B}_{\alpha}(\bar{y}) \text { and } w, w^{\prime} \in \mathbb{B}_{\alpha}(\bar{w})
$$

The next result is taken from $[1$, Theorem 5.1$]$.

Theorem 2.2 (Lipschitz-like property of solution maps via metric regularity of fields in generalized equations). Let $f: X \times Y \rightarrow Z$ be a mapping between Banach spaces that is Lipschitz continuous on a neighborhood $U \times V$ of $(\bar{x}, \bar{y}) \in X \times Y$, and let $Q: Y \rightrightarrows Z$ be a set-valued field mapping with $\bar{z}:=-f(\bar{x}, \bar{y}) \in Q(\bar{y})$ such that the graph of $Q$ is locally closed around $(\bar{y}, \bar{z})$. The following assertions hold:

(i) Assume that $A \in \mathcal{L}(X, Z)$ is a surjective linear operator satisfying (2.6) with some $\mu \geq 0$. If the solution map $S: X \rightrightarrows Y$ in (1.2) is Lipschitz-like around $(\bar{x}, \bar{y})$ and if the condition

$$
\operatorname{reg} A \cdot\left[\mu+\operatorname{lip} S(\bar{x}, \bar{y}) \cdot \widehat{\operatorname{lip}}_{y} f(\bar{x}, \bar{y})\right]<1
$$


is fulfilled, then $Q$ is metrically regular around $(\bar{y}, \bar{z})$ with the exact bound estimate

$$
\operatorname{reg} Q(\bar{y}, \bar{z}) \leq \frac{\operatorname{lip} S(\bar{x}, \bar{y}) \cdot \operatorname{reg} A}{1-\operatorname{reg} A \cdot\left[\mu+\operatorname{lip} S(\bar{x}, \bar{y}) \cdot \widehat{\operatorname{lip}}_{y} f(\bar{x}, \bar{y})\right]} .
$$

(ii) Conversely, assume that $Q$ is metrically regular around $(\bar{y}, \bar{z})$ and that the condition

$$
\widehat{\operatorname{lip}}_{y} f(\bar{x}, \bar{y}) \cdot \operatorname{reg} Q(\bar{y}, \bar{z})<1
$$

is satisfied. Then $S$ is Lipschitz-like around $(\bar{x}, \bar{y})$ with the exact bound estimate

$$
\operatorname{lip} S(\bar{x}, \bar{y}) \leq \frac{\operatorname{reg} Q(\bar{y}, \bar{z}) \cdot \widehat{\operatorname{lip}}_{x} f(\bar{x}, \bar{y})}{1-\operatorname{reg} Q(\bar{y}, \bar{z}) \cdot \widehat{\operatorname{lip}}_{y} f(\bar{x}, \bar{y})} .
$$

The following well known result on the preservation of metric regularity under Lipschitzian perturbations can be proved as a direct consequence of assertion (ii) of Theorem 2.2 by taking $f(x, y)=-x+g(y)$ and $Q=F$.

Theorem 2.3 (metric regularity under Lipschitzian perturbations). Let $F: X \rightrightarrows Y$ be $a$ set-valued mapping between Banach spaces with locally closed graph around $(\bar{x}, \bar{y}) \in \operatorname{gph} F$. Assume that $F$ be metrically regular around $(\bar{x}, \bar{y})$ with constant $\kappa>0$ and consider a single-valued mapping $g: X \rightarrow Y$ Lipschitz continuous around $\bar{x}$ with constant $\lambda \geq 0$. satisfying $\lambda<\kappa^{-1}$. Then $F+g$ is metrically regular around $(\bar{x}, \bar{y}+g(\bar{x}))$ with constant $\kappa /(1-\kappa \lambda)$.

\section{Partial metric regularity and its applications}

In this section we introduce the notion of partial metric regularity and apply it to establishing various extensions of the aforementioned results from [1].

Definition 3.1 (partial metric regularity). A set-valued mapping $F: X \times Y \rightrightarrows Z$ is said to be METRICALLY REGULAR WITH RESPECT TO $x$ UNIFORMLY IN $y$ AROUND $((\bar{x}, \bar{y}), \bar{z}) \in \operatorname{gph} F$ if there are neighborhoods $U$ of $\bar{x}, V$ of $\bar{y}$, and $W$ of $\bar{z}$ as well as a constant $\kappa>0$ such that

$$
d\left(x, F^{-1}(\cdot, y)(z)\right) \leq \kappa d(z, F(x, y)) \text { for all } x \in U, y \in V \text { and } z \in W
$$

where $F^{-1}(\cdot, y)(z)=\{x \in X \mid z \in F(x, y)\}$. The infimum of $\kappa>0$ over all the combinations $(\kappa, U, V, W)$ for which (3.1) holds is called the EXACT PARTIAL UNIFORM REGULARITY BOUND of $F$ in $x$ around $(\bar{x}, \bar{y})$ and is denoted $\widehat{\operatorname{reg}}_{x} F((\bar{x}, \bar{y}), \bar{z})$.

Observe that a mapping $F: X \times Y \rightrightarrows Z$ is metrically regular around $((\bar{x}, \bar{y}), \bar{z})$ if $F$ is metrically regular with respect to $x$ uniformly in $y$ around this point, since

$$
d\left((x, y), F^{-1}(z)\right) \leq d\left(x, F^{-1}(\cdot, y)(z)\right) .
$$

By symmetry we can define the metric regularity of $F: X \times Y \rightrightarrows Z$ with respect to $y$ uniformly in $x$ around $((\bar{x}, \bar{y}), \bar{z}) \in \operatorname{gph} F$ and its exact bound $\widehat{\operatorname{reg}}_{y} F((\bar{x}, \bar{y}), \bar{z})$ and make the same observation.

The next result provides sufficient conditions for the partial metric regularity with an upper estimate of the exact regularity bound. 
Proposition 3.2 (sufficient conditions for partial metric regularity). Let $f: X \times Y \rightarrow Z$ be a mapping between Banach spaces continuous at $(\bar{x}, \bar{y}) \in X \times Y$, and let $\bar{z}:=f(\bar{x}, \bar{y})$. Given a surjective linear operator $A \in \mathcal{L}(X, Z)$, suppose that there are neighborhoods $U$ of $\bar{x}$ and $V$ of $\bar{y}$ and a number $\mu \geq 0$ such that $\mu \cdot \operatorname{reg} A<1$ and condition (2.6) holds. Then $f$ is metrically regular with respect to $x$ uniformly in $y$ around $((\bar{x}, \bar{y}), \bar{z})$ with the following upper estimate of the exact bound:

$$
\widehat{\operatorname{reg}}_{x} f(\bar{x}, \bar{y}) \leq \frac{\operatorname{reg} A}{1-\mu \cdot \operatorname{reg} A} .
$$

Proof. Pick a number $\gamma>\operatorname{reg} A$ with $\mu \gamma<1$, take $g(z):=-z$, and apply Theorem 2.1 having in mind inclusion (2.10). In this way we find a constant $\alpha>0$ such that

$$
\Gamma\left(y, z^{\prime}\right) \cap \mathbb{B}_{\alpha}(\bar{x}) \subset \Gamma(y, z)+\frac{\gamma}{1-\gamma \mu}\left\|z-z^{\prime}\right\| \mathbb{B} \text { for all } y \in \mathbb{B}_{\alpha}(\bar{y}) \text { and } z, z^{\prime} \in \mathbb{B}_{\alpha}(\bar{z})
$$

where $\Gamma(y, z):=\{x \in X \mid f(x, y)=z\}$. By the continuity of $f$ at $(\bar{x}, \bar{y})$ we get a positive number $\beta$ with $\beta \leq \alpha$ for which

$$
\|f(x, y)-\bar{z}\| \leq \alpha \text { whenever }(x, y) \in \mathbb{B}_{\beta}(\bar{x}) \times \mathbb{B}_{\beta}(\bar{y}) .
$$

Fix further $x \in \mathbb{B}_{\beta}(\bar{x}), y \in \mathbb{B}_{\beta}(\bar{y})$, and $z \in \mathbb{B}_{\alpha}(\bar{z})$. Since $x \in \Gamma(y, f(x, y)) \cap \mathbb{B}_{\alpha}(\bar{x})$, there is $x^{\prime} \in \Gamma(y, z)$ satisfying the estimate

$$
\left\|x-x^{\prime}\right\| \leq \frac{\gamma}{1-\gamma \mu} \| z-f(x, y) \Uparrow .
$$

Thus we arrive at the inequality

$$
d\left(x, f^{-1}(\cdot, y)(z)\right) \leq\left\|x-x^{\prime}\right\| \leq \frac{\gamma}{1-\gamma \mu}\|z-f(x, y)\|,
$$

which clearly implies the metric regularity of $f$ with respect to $x$ uniformly in $y$ around $((\bar{x}, \bar{y}), \bar{z})$ with constant $\gamma /(1-\gamma \mu)$. Since $\gamma>0$ was chosen arbitrarily close to reg $A$, we get the upper estimate (3.2) and complete the proof of the proposition.

Remark 3.3 (partial metric regularity for nonsmooth functions). There are examples of mappings that are metrically regular with respect to $x$ uniformly in $y$ around some point but such that they do not satisfy the hypotheses of Proposition 3.2. For instance, consider the real-valued function $f: \mathbb{R} \times \mathbb{R} \rightarrow \mathbb{R}$ defined by

$$
f(x, y)= \begin{cases}\sqrt{x}+y & \text { for } x \geq 0 \\ -\sqrt{-x}+y, & \text { for } x<0\end{cases}
$$

It is easy to check that this function is metrically regular with respect to $x$ uniformly in $y$ around the origin while for any linear operator $A \in \mathcal{L}(\mathbb{R}, \mathbb{R})$ we have $\widehat{\operatorname{lip}}_{x} g(0,0)=\infty$ for $g(x, y):=f(x, y)-A x$.

The phenomenon observed in Remark 3.3 is due to the nonsmoothness of the function under consideration. For (partially) strictly differentiable mappings we can take by $A$ the corresponding partial derivative and show that the partial metric regularity of $f$ reduces in fact to the usual metric regularity of the partial derivative around the point in question. Recall that a mapping $f: X \times Y \rightarrow Z$ is strictly partially differentiable at $(\bar{x}, \bar{y})$ with respect to $x$ uniformly in $y$ with the partial derivative $\nabla_{x} f(\bar{x}, \bar{y})$ if

$$
\lim _{\substack{x, x^{\prime} \rightarrow \bar{x} \\ x \neq x^{\prime}}} \frac{f(x, y)-f\left(x^{\prime}, y\right)-\left\langle\nabla_{x} f(\bar{x}, \bar{y}), x-x^{\prime}\right\rangle}{\left\|x-x^{\prime}\right\|}=0 \text { for all } y \in Y \text { near } \bar{y}
$$


Proposition 3.4 (partial metric regularity of partially smooth mappings). Consider a mapping $f: X \times Y \rightarrow Z$ between Banach spaces, and let $(\bar{x}, \bar{y}) \in X \times Y$ be such that $f$ is continuous at $(\bar{x}, \bar{y})$ and strictly partially differentiable at this point with respect to $x$ uniformly in $y$. Assume that the partial derivative operator $\nabla_{x} f(\bar{x}, \bar{y}): X \rightarrow Z$ is surjective. Then we have

$$
\widehat{\operatorname{reg}}_{x} f(\bar{x}, \bar{y})=\operatorname{reg} \nabla_{x} f(\bar{x}, \bar{y})=\left\|\left(\nabla_{x} f(\bar{x}, \bar{y})^{*}\right)^{-1}\right\| .
$$

Proof. The second equality in (3.4) follows from the well-known fact (see, e.g., [4, Corollary 1.58]) that a linear bounded operator $A \in \mathcal{L}(X, Y)$ is metrically regular around every point $x \in X$ if and only if it is surjective; in this case the exact regularity bound of $A$ is computed by

$$
\operatorname{reg} A=\left\|\left(A^{*}\right)^{-1}\right\| \text {. }
$$

Further, it is easy to get from Proposition 3.2 that

$$
\widehat{\operatorname{reg}}_{x} f(\bar{x}, \bar{y}) \leq \operatorname{reg} \nabla_{x} f(\bar{x}, \bar{y}) \text {. }
$$

On the other hand, the strict partial differentiability of $f$ with respect to $x$ ensures the equality

$$
\operatorname{lip}\left(f(\cdot, \bar{y})-\nabla_{x} f(\bar{x}, \bar{y})\right)(\bar{x})=0
$$

Employing finally Theorem 2.3 , we conclude that

$$
\operatorname{reg} \nabla_{x} f(\bar{x}, \bar{y})=\operatorname{reg} f(\cdot, \bar{y})(\bar{x}) \leq \widehat{\operatorname{reg}}_{x} f(\bar{x}, \bar{y}),
$$

which justifies (3.4) and thus completes the proof of the proposition.

Having in mind the results of Proposition 3.2 and Remark 3.3, we obtain now the following extension of Theorem 2.1 on Lipschitzian behavior of implicit multifunctions.

Theorem 3.5 (Lipschitzian properties of implicit multifunctions under partial metric regularity). Let $f: X \times Y \rightarrow Z$ be a mapping between Banach spaces, and let $(\bar{x}, \bar{y}) \in X \times Y$ be such that $f(\cdot, y)$ is continuous around $\bar{x}$ for each $y$ around $\bar{y}$. Given a mapping $g: W \rightarrow Z$ between Banach spaces with $g(\bar{w})=-f(\bar{x}, \bar{y})$ for some $\bar{w} \in W$, consider a set-valued mapping $\Gamma: Y \times W \Rightarrow X$ (implicit multifunction) defined in (2.7). Assume further that $f$ is metrically regular with respect to $x$ uniformly in $y$ around $(\bar{x}, \bar{y})$ with constant $\kappa>0$, that $f$ is locally Lipschitzian with respect to $y$ with constant $\eta \geq 0$ uniformly in $x$ around $(\bar{x}, \bar{y})$, and that $g$ is locally Lipschitzian around $\bar{w} \in W$ with constant $\lambda$. Then there is $\alpha>0$ such that for every $(y, w),\left(y^{\prime}, w^{\prime}\right) \in \mathbb{B}_{\alpha}(\bar{y}) \times$ $\mathbb{B}_{\alpha}(\bar{w})$ we have the inclusion

$$
\Gamma\left(y^{\prime}, w^{\prime}\right) \cap \mathbb{B}_{\alpha}(\bar{x}) \subset \Gamma(y, w)+\kappa\left(\eta\left\|y-y^{\prime}\right\|+\lambda\left\|w-w^{\prime}\right\|\right) \mathbb{B} .
$$

The latter implies that $\Gamma$ is Lipschitz-like around $((\bar{y}, \bar{w}), \bar{x})$ and that its exact Lipschitzian bound satisfies the upper estimate

$$
\operatorname{lip} \Gamma((\bar{y}, \bar{w}), \bar{x}) \leq \widehat{\operatorname{reg}}_{x} f(\bar{x}, \bar{y}) \cdot \max \left\{\widehat{\operatorname{lip}}_{y} f(\bar{x}, \bar{y}), \operatorname{lip} g(\bar{w})\right\} .
$$

Proof. Taking a positive constant $a$ such that the mapping $x \mapsto f(x, y)$ is continuous on $\mathbb{B}_{a}(\bar{x})$ for every $y \in \mathbb{B}_{a}(\bar{y})$, we have

$$
\begin{gathered}
\left\|g(w)-g\left(w^{\prime}\right)\right\| \leq \lambda\left\|w-w^{\prime}\right\| \text { for all } w, w^{\prime} \in \mathbb{B}_{a}(\bar{w}) \\
\left\|f(x, y)-f\left(x, y^{\prime}\right)\right\| \leq \eta\left\|y-y^{\prime}\right\| \text { for all } x \in \mathbb{B}_{a}(\bar{x}) \text { and } y, y^{\prime} \in \mathbb{B}_{a}(\bar{y}), \\
d\left(x, f^{-1}(\cdot, y)(z)\right) \leq \kappa\|z-f(x, y)\| \text { for all } x \in \mathbb{B}_{a}(\bar{x}), y \in \mathbb{B}_{a}(\bar{y}) \text { and } z \in \mathbb{B}_{a}(f(\bar{x}, \bar{y})) .
\end{gathered}
$$


Further, let $0<\alpha \leq a$ be such that $\lambda \alpha \leq a$. Pick $(y, w),\left(y^{\prime}, w^{\prime}\right) \in \mathbb{B}_{\alpha}(\bar{y}) \times \mathbb{B}_{\alpha}(\bar{w})$ and then take $x^{\prime} \in \Gamma\left(y^{\prime}, w^{\prime}\right) \cap \mathbb{B}_{\alpha}(\bar{x})$. We get

$$
\|-g(w)-f(\bar{x}, \bar{y})\| \leq \lambda\|w-\bar{w}\| \leq \lambda \alpha \leq a,
$$

which implies the estimates

$$
\begin{aligned}
d\left(x^{\prime}, f^{-1}(\cdot, y)(-g(w))\right) & \leq \kappa\left\|f\left(x^{\prime}, y\right)+g(w)\right\| \leq \kappa\left(\left\|f\left(x^{\prime}, y\right)-f\left(x^{\prime}, y^{\prime}\right)\right\|+\left\|g(w)-g\left(w^{\prime}\right)\right\|\right) \\
& \leq \kappa\left(\eta\left\|y-y^{\prime}\right\|+\lambda\left\|w-w^{\prime}\right\|\right) .
\end{aligned}
$$

From the continuity of $f$ we obviously have the closedness of the inverse image $f^{-1}(\cdot, y)(-g(w))$, and hence there is $x \in \Gamma(y, w)$ such that

$$
\left\|x-x^{\prime}\right\| \leq \kappa\left(\eta\left\|y-y^{\prime}\right\|+\lambda\left\|w-w^{\prime}\right\|\right) .
$$

The latter yields the estimate (3.7) and thus completes the proof of the theorem.

Using the new implicit multifunction result of Theorem 3.5 instead of the one of Theorem 2.1, we can extend several relationships between metric regularity and Lipschitzian properties in the framework of generalized equations (1.1) established in [1]. In particular, we get the following equivalencies under milder assumptions in comparison with [1, Theorem 3.3].

Theorem 3.6 (metric regularity of solution maps via Lipschitzian properties of fields in generalized equations). Let $f: X \times Y \rightarrow Z$ be a mapping between Banach spaces, and let $(\bar{x}, \bar{y}) \in X \times Y$ be such that $f$ is Lipschitz continuous on some neighborhood of $(\bar{x}, \bar{y})$. Assume also that $f$ is metrically regular with respect to $x$ uniformly in $y$ around $(\bar{x}, \bar{y})$. Let $Q: Y \rightrightarrows Z$ be a set-valued field mapping with $\bar{z}:=-f(\bar{x}, \bar{y}) \in Q(\bar{y})$. Then the following assertions are satisfied:

(i) The solution map $S$ in (1.2) is metrically regular around $(\bar{x}, \bar{y})$ if and only if the field $Q$ in (1.1) is Lipschitz-like around $(\bar{y}, \bar{z})$. Moreover, we have the exact bound relationships

$$
\begin{gathered}
\operatorname{reg} S(\bar{x}, \bar{y}) \leq \widehat{\operatorname{reg}}_{x} f(\bar{x}, \bar{y}) \cdot\left[\operatorname{lip} Q(\bar{y}, \bar{z})+\widehat{\operatorname{lip}}_{y} f(\bar{x}, \bar{y})\right] \\
\operatorname{lip} Q(\bar{y}, \bar{z}) \leq \widehat{\operatorname{lip}}_{x} f(\bar{x}, \bar{y}) \cdot \operatorname{reg} S(\bar{x}, \bar{y})+\widehat{\operatorname{lip}}_{y} f(\bar{x}, \bar{y})
\end{gathered}
$$

(ii) The solution map $S$ is metrically subregular at $(\bar{x}, \bar{y})$ if and only if the field $Q$ is calm at $(\bar{y}, \bar{z})$. Furthermore, we have the exact bound relationships

$$
\begin{gathered}
\text { subreg } S(\bar{x}, \bar{y}) \leq \widehat{\operatorname{reg}}_{x} f(\bar{x}, \bar{y}) \cdot\left[\operatorname{clm} Q(\bar{y}, \bar{z})+\widehat{\operatorname{lip}}_{y} f(\bar{x}, \bar{y})\right] \\
\operatorname{clm} Q(\bar{y}, \bar{z}) \leq \widehat{\operatorname{lip}}_{x} f(\bar{x}, \bar{y}) \cdot \operatorname{subreg} S(\bar{x}, \bar{y})+\widehat{\operatorname{lip}}_{y} f(\bar{x}, \bar{y})
\end{gathered}
$$

Proof. Follows that of [1, Theorem 3.3] by using Theorem 3.5 instead of Theorem 2.1.

The next theorem provides extensions of the results in [1] establishing relationships between Lipschitzian properties of solutions maps and metric regularity of field mappings in systems (1.2).

Theorem 3.7 (Lipschitz-like property of solution maps via metric regularity of fields in generalized equations). Let $f: X \times Y \rightarrow Z$ be a mapping between Banach spaces, and let $(\bar{x}, \bar{y}) \in X \times Y$ be such that $f$ is locally Lipschitzian with respect to $y$ uniformly in $x$ on some neighborhood $U \times V$ of $(\bar{x}, \bar{y})$, and let $f(\cdot, y)$ be continuous on $U$ for each $y \in V$. Assume also that $f$ is metrically regular with respect to $x$ uniformly in $y$ around $(\bar{x}, \bar{y})$. Let $Q: Y \Rightarrow Z$ be a set-valued 
field mapping with $\bar{z}:=-f(\bar{x}, \bar{y}) \in Q(\bar{y})$ such that the graph of $Q$ is locally closed around $(\bar{y}, \bar{z})$. If the solution map $S: X \Rightarrow Y$ in (1.2) is Lipschitz-like around $(\bar{x}, \bar{y})$ and if the condition

$$
\widehat{\operatorname{reg}}_{x} f(\bar{x}, \bar{y}) \cdot \widehat{\operatorname{lip}}_{y} f(\bar{x}, \bar{y}) \cdot \operatorname{lip} S(\bar{x}, \bar{y})<1
$$

is fulfilled, then $Q$ is metrically regular around $(\bar{y}, \bar{z})$ with the exact bound estimate

$$
\operatorname{reg} Q(\bar{y}, \bar{z}) \leq \frac{\widehat{\operatorname{reg}}_{x} f(\bar{x}, \bar{y}) \cdot \operatorname{lip} S(\bar{x}, \bar{y})}{1-\widehat{\operatorname{reg}}_{x} f(\bar{x}, \bar{y}) \cdot \widehat{\operatorname{lip}}_{y} f(\bar{x}, \bar{y}) \cdot \operatorname{lip} S(\bar{x}, \bar{y})}
$$

Proof. Follows that of [1, Theorem 5.1] with using the improved implicit multifunction result of Theorem 3.5 instead of the one in Theorem 2.1.

Now we establish a converse statement to Theorem 3.5, which derives the partial metric regularity of the base mapping $f$ in (2.7) from the (partial) Lipschitz-like property of the implicit multifunction $\Gamma$ around the corresponding points.

Theorem 3.8 (partial metric regularity of base mappings from Lipschitzian properties of implicit multifunctions). Let $f: X \times Y \rightarrow Z$ be a mapping between Banach spaces continuous at $(\bar{x}, \bar{y}) \in X \times Y$. Given a mapping $g: W \rightarrow Z$ between Banach spaces such that $g(\bar{w})=-f(\bar{x}, \bar{y})$ for some $\bar{w} \in W$, assume that $g$ is metrically regular around $(\bar{w}, g(\bar{w}))$. Suppose also that the implicit multifunction $\Gamma$ defined in (2.7) is Lipschitz-like with respect to $w$ uniformly in $y$ around $((\bar{y}, \bar{w}), \bar{x})$. Then $f$ is metrically regular with respect to $x$ uniformly in $y$ around $(\bar{x}, \bar{y})$ with the following upper estimate of the exact partial regularity bound:

$$
\widehat{\operatorname{reg}}_{x} f(\bar{x}, \bar{y}) \leq \widehat{\operatorname{lip}}_{w} \Gamma((\bar{y}, \bar{w}), \bar{x}) \cdot \operatorname{reg} g(\bar{w})
$$

Proof. Take any $\ell>\widehat{\operatorname{lip}}_{w} \Gamma((\bar{y}, \bar{w}), \bar{x})$ and $\kappa>\operatorname{reg} g(\bar{w})$ and then pick $\alpha>0$ such that

$$
\begin{gathered}
d\left(w, g^{-1}(z)\right) \leq \kappa\|z-g(w)\| \text { and } \\
\Gamma(y, w) \cap \mathbb{B}_{\alpha}(\bar{x}) \subset \Gamma\left(y, w^{\prime}\right)+\ell\left\|w-w^{\prime}\right\| \mathbb{B}
\end{gathered}
$$

for every $y \in \mathbb{B}_{\alpha}(\bar{y}), w, w^{\prime} \in \mathbb{B}_{\alpha}(\bar{w})$, and $z \in \mathbb{B}_{\alpha}(g(\bar{w}))$. Select further a constant $0<a \leq \alpha$ with

$$
(\kappa+1)(a+2\|f(x, y)-f(\bar{x}, \bar{y})\|) \leq \alpha \text { whenever } x \in \mathbb{B}_{a}(\bar{x}), y \in \mathbb{B}_{a}(\bar{y}) .
$$

For each $0<\varepsilon<1$ we fix $x \in \mathbb{B}_{a}(\bar{x}), y \in \mathbb{B}_{a}(\bar{y})$, and $z \in \mathbb{B}_{a}(f(\bar{x}, \bar{y}))$. It follows from (3.10) that $-f(x, y) \in \mathbb{B}_{\alpha}(g(\bar{w}))$, and thus there is $w \in g^{-1}(-f(x, y))$ satisfying

$$
\|w-\bar{w}\| \leq(\kappa+\varepsilon)\|-f(x, y)-g(\bar{w})\| \leq \alpha .
$$

By taking the inclusion $-z \in \mathbb{B}_{\alpha}(-f(\bar{x}, \bar{y}))=\mathbb{B}_{\alpha}(g(\bar{w}))$ into account, we find $w^{\prime} \in g^{-1}(-z)$ with

$$
\left\|w-w^{\prime}\right\| \leq(\kappa+\varepsilon)\|-z-g(w)\|=(\kappa+\varepsilon)\|z-f(x, y)\| .
$$

The latter implies the estimates

$$
\begin{aligned}
\left\|w^{\prime}-\bar{w}\right\| & \leq\left\|w^{\prime}-w\right\|+\|w-\bar{w}\| \leq(\kappa+\varepsilon)(\|z-f(x, y)\|+\|f(x, y)-f(\bar{x}, \bar{y})\|) \\
& \leq(\kappa+\varepsilon)(a+2\|f(x, y)-f(\bar{x}, \bar{y})\|) \leq \alpha .
\end{aligned}
$$


It now follows from $x \in \Gamma(y, w) \cap \mathbb{B}_{\alpha}(\bar{x})$ that there is $x^{\prime} \in \Gamma\left(y, w^{\prime}\right)$ satisfying

$$
\left\|x-x^{\prime}\right\| \leq \ell\left\|w-w^{\prime}\right\| \leq(\kappa+\varepsilon) \ell\|z-f(x, y)\| .
$$

Remembering that the positive numbers $\varepsilon, \kappa$, and $\ell$ were chosen to be arbitrarily close to zero, $\operatorname{reg} g(\bar{w})$, and $\widehat{\operatorname{lip}}_{w} \Gamma((\bar{y}, \bar{w}), \bar{x})$, respectively, we complete the proof of the theorem.

Next obtain the following specifications of the results above in the case of (partially) strictly differentiable mappings $f$ and $g$ in the framework of implicit multifunctions (2.7).

Proposition 3.9 (implicit multifunctions in partially smooth settings). Let $f: X \times Y \rightarrow Z$ be a mapping between Banach spaces, and let $(\bar{x}, \bar{y}) \in X \times Y$ be such that $f$ is locally Lipschitzian around $(\bar{x}, \bar{y})$ and strictly partially differentiable at this point with respect to $x$ uniformly in $y$. Let further $g: W \rightarrow Z$ be a mapping between Banach spaces strictly differentiable at $\bar{w} \in W$ with the surjective derivative $\nabla g(\bar{w})$ and such that $g(\bar{w})=-f(\bar{x}, \bar{y})$. Then the set-valued mapping $\Gamma: Y \times W \Rightarrow X$ defined by (2.7) is Lipschitz-like around $((\bar{y}, \bar{w}), \bar{x})$ if and only if the partial derivative operator $\nabla_{x} f(\bar{x}, \bar{y})$ is surjective. In this case we have the relationships

$$
\begin{gathered}
\widehat{\operatorname{lip}}_{y} \Gamma((\bar{y}, \bar{w}), \bar{x}) \leq\left\|\left(\nabla_{x} f(\bar{x}, \bar{y})^{*}\right)^{-1}\right\| \cdot\left\|\nabla_{y} f(\bar{x}, \bar{y})\right\|, \\
\widehat{\operatorname{lip}}_{w} \Gamma((\bar{y}, \bar{w}), \bar{x}) \leq\left\|\left(\nabla_{x} f(\bar{x}, \bar{y})^{*}\right)^{-1}\right\| \cdot\|\nabla g(\bar{w})\|, \\
\widehat{\operatorname{reg}}_{x} f(\bar{x}, \bar{y})=\left\|\left(\nabla_{x} f(\bar{x}, \bar{y})^{*}\right)^{-1}\right\| \leq \widehat{\operatorname{lip}}_{w} \Gamma((\bar{y}, \bar{w}), \bar{x}) \cdot\left\|\left(\nabla g(\bar{w})^{*}\right)^{-1}\right\| .
\end{gathered}
$$

Proof. This follows directly from of Theorem 3.5, Theorem 3.8, and Proposition 3.4.

Define now the relative condition number of $F: X \rightrightarrows Y$ at $(\bar{x}, \bar{y}) \in \operatorname{gph} F$ by

$$
C(F(\bar{x}, \bar{y})):=\operatorname{reg} F(\bar{x}, \bar{y}) \cdot \operatorname{lip} F(\bar{x}, \bar{y})=\operatorname{reg} F(\bar{x}, \bar{y}) \cdot \operatorname{reg} F^{-1}(\bar{y}, \bar{x})
$$

with the convention that $C(F(\bar{x}, \bar{y})):=\infty$ when either $F$ or $F^{-1}$ is not metrically regular around the point. It follows from definition (3.11) and [2, Exercise 3E.11] that $C(F(\bar{x}, \bar{y})) \geq 1$ when $(\bar{x}, \bar{y}) \notin \operatorname{int} g p h F$. The reader is referred to [5] for more information on condition numbers for single-valued mappings and their applications to numerical aspects of optimization.

Corollary 3.10 (precise formulas for exact bounds). Under the assumptions of Proposition (3.9) we have the equalities

$$
\widehat{\operatorname{lip}}_{w} \Gamma((\bar{y}, \bar{w}), \bar{x})=\operatorname{reg} \nabla_{x} f(\bar{x}, \bar{y}) \cdot \operatorname{lip} g(\bar{w})=\left\|\left(\nabla_{x} f(\bar{x}, \bar{y})^{*}\right)^{-1}\right\| \cdot\|\nabla g(\bar{w})\|
$$

provided that the relative condition number of $g: W \rightarrow Z$ at $\bar{w}$ is

$$
C(g(\bar{w}))=\|\nabla g(\bar{w})\| \cdot\left\|\left(\nabla g(\bar{w})^{*}\right)^{-1}\right\|=1 .
$$

In particular, for $g(z):=-z$ and $f: X \rightarrow Y$ satisfying $\widehat{\operatorname{lip}}_{y} f(\bar{x}, \bar{y}) \leq 1$ we get the relationship

$$
\operatorname{lip} \Gamma(\bar{y}, \bar{x})=\operatorname{reg} \nabla_{x} f(\bar{x}, \bar{y}) .
$$

Proof. Both equalities in (3.12) follow from the estimates of Proposition 3.9 and definition (3.11) under assumption (3.13) on the relative condition number of the smooth mapping $g$. This immediately implies (3.14) in the particular case under consideration. 


\section{Strong regularity/subregularity and Lipschitzian localization}

In this section we study the notion of strong regularity (known also as strong metric regularity) introduced by Robinson [6] for variational inequalities and then widely applied in many publications to sensitivity analysis and numerical methods for optimization-related and equilibrium problems. In parallel we pay attention to the corresponding notion of strong subregularity; see [2] and the references therein. Our main results in this section concern qualitative and quantitative relations between strong metric regularity/subregularity and single-valued Lipschitzian/calmness localizations in the framework of the parametric variational systems (1.1).

Recall that a mapping $F: X \rightrightarrows Y$ is strongly metrically regular (or just strongly regular) around $(\bar{x}, \bar{y})$ with constant $\kappa>0$ if there are neighborhoods $U \subset X$ of $\bar{x}$ and $V \subset Y$ of $\bar{y}$ such that the set $F^{-1}(y) \cap U$ is singleton for every $y \in V$ and that

$$
d\left(x, F^{-1}(y)\right) \leq \kappa d(y, F(x)) \text { for all } x \in U \text { and } y \in V .
$$

A mapping $F: X \Rightarrow Y$ is strongly metrically subregular (or just strongly subregular) at $(\bar{x}, \bar{y})$ with constant $\kappa>0$ if there is a neighborhood $U$ of $\bar{x}$ such that

$$
\|x-\bar{x}\| \leq \kappa d(\bar{y}, F(x)) \text { for all } x \in U
$$

We say as usual that a set-valued mapping admits a single-valued localization around some point if there is a neighborhood of this point where the mapping is actually single-valued. It follows from the well-known equivalence between metric regularity (resp. subregularity) of $F$ and the Lipschitzlike (resp. calmness) property of $F^{-1}$ and the definitions above that this line of equivalence also holds between the strong versions of metric regularity/subregularity of arbitrary mappings $F$ and the corresponding single-valued Lipschitzian localizations of their inverses.

The next result establishes two-sided qualitative and quantitative relationships between the single-valued Lipschitzian localization of the solution map (1.2) and the strong regularity of the field in the generalized equation(1.1) under appropriate assumptions.

Theorem 4.1 (relationships between single-valued Lipschitzian localization of solution maps and strong regularity of fields in generalized equations). Let $f: X \times Y \rightarrow Z$ be a mapping between Banach spaces, let $(\bar{x}, \bar{y}) \in X \times Y$, and let $Q: Y \Rightarrow Z$ be a set-valued field mapping in (1.1) with $\bar{z}:=-f(\bar{x}, \bar{y}) \in Q(\bar{y})$ such that the graph of $Q$ is locally closed around $(\bar{y}, \bar{z})$. The following assertions hold:

(i) Assume that $f$ is locally Lipschitzian with respect to $y$ uniformly in $x$ on some neighborhood $U \times V$ of $(\bar{x}, \bar{y})$, and let $f(\cdot, y)$ be continuous on $U$ for each $y \in V$. Suppose also that $f$ is metrically regular with respect to $x$ uniformly in $y$ around $(\bar{x}, \bar{y})$. If the solution map $S: X \rightrightarrows Y$ in (1.2) admits a single-valued Lipschitzian localization around $(\bar{x}, \bar{y})$ and if condition (3.8) is satisfied, then $Q$ is strongly metrically regular around $(\bar{y}, \bar{z})$ with the exact bound upper estimate

$$
\operatorname{reg} Q(\bar{y}, \bar{z}) \leq \frac{\widehat{\operatorname{reg}}_{x} f(\bar{x}, \bar{y}) \cdot \operatorname{lip} S(\bar{x}, \bar{y})}{1-\widehat{\operatorname{reg}}_{x} f(\bar{x}, \bar{y}) \cdot \widehat{\operatorname{lip}}_{y} f(\bar{x}, \bar{y}) \cdot \operatorname{lip} S(\bar{x}, \bar{y})}
$$

(ii) Conversely, assume that $f$ is Lipschitz around $(\bar{x}, \bar{y})$, that $Q$ is strongly metrically regular around $(\bar{y}, \bar{z})$, and that condition

$$
\widehat{\operatorname{lip}}_{y} f(\bar{x}, \bar{y}) \cdot \operatorname{reg} Q(\bar{y}, \bar{z})<1
$$


is satisfied. Then the solution map $S$ admits a single-valued Lipschitzian localization around $(\bar{x}, \bar{y})$ with the exact bound estimate

$$
\operatorname{lip} S(\bar{x}, \bar{y}) \leq \frac{\operatorname{reg} Q(\bar{y}, \bar{z}) \cdot \widehat{\operatorname{lip}}_{x} f(\bar{x}, \bar{y})}{1-\operatorname{reg} Q(\bar{y}, \bar{z}) \cdot \widehat{\operatorname{lip}}_{y} f(\bar{x}, \bar{y})} .
$$

Proof. To justify assertion (i), choose $\ell>\operatorname{lip} S(\bar{x}, \bar{y}), \kappa>\widehat{\operatorname{reg}}_{x} f(\bar{x}, \bar{y})$, and $\eta_{y}>\widehat{\operatorname{lip}}_{y} f(\bar{x}, \bar{y})$ with $\ell \kappa \eta_{y}<1$. Then find a positive constant $\alpha$ and a mapping $s: X \rightarrow Y$ such that $s(x)=S(x) \cap \mathbb{B}_{\alpha}(\bar{y})$ for $x \in \mathbb{B}_{\alpha}(\bar{x})$ and that

$$
\left\|s(x)-s\left(x^{\prime}\right)\right\| \leq \ell\left\|x-x^{\prime}\right\| \text { for all } x, x^{\prime} \in \mathbb{B}_{\alpha}(\bar{x}) .
$$

By Theorem 3.5 with $\Gamma(y, z):=\{x \in X \mid f(x, y)+z=0\}$ we can make $\alpha>0$ smaller if necessary to ensure the inclusion

$$
\Gamma\left(y^{\prime}, z^{\prime}\right) \cap \mathbb{B}_{\boldsymbol{\alpha}}(\bar{x}) \subset \Gamma(y, z)+\kappa\left(\eta_{y}\left\|y-y^{\prime}\right\|+\left\|z-z^{\prime}\right\|\right) \mathbb{B}
$$

for all $(y, z),\left(y^{\prime}, z^{\prime}\right) \in \mathbb{B}_{\alpha}(\bar{y}) \times \mathbb{B}_{\alpha}(\bar{z})$. On the other hand, it follows from Theorem 3.7 that $Q$ is metrically regular around $(\bar{y}, \bar{z})$ with the exact bound estimate (2.11). Hence it remains to prove that $Q^{-1}$ admits a single-valued localization.

To proceed, pick a positive constant $a \leq \alpha$ for which we have the condition

$$
\left(3 \eta_{y}+1\right) \kappa a \leq \alpha .
$$

Suppose further that $y, y^{\prime} \in Q^{-1}(z) \cap \mathbb{B}_{a}(\bar{y})$ for some $z \in \mathbb{B}_{a}(\bar{z})$. Then by (4.6) there is some $x \in \Gamma(y, z)$ satisfying the estimates

$$
\|x-\bar{x}\| \leq \kappa\left(\eta_{y}\|y-\bar{y}\|+\|z-\bar{z}\|\right) \leq\left(\eta_{y}+1\right) \kappa a \leq \alpha,
$$

which give $x \in \Gamma(y, z) \cap \mathbb{B}_{\alpha}(\bar{x})$. Employing (4.6) again, we find $x^{\prime} \in \Gamma\left(y^{\prime}, z\right)$ such that

$$
\left\|x-x^{\prime}\right\| \leq \kappa \eta_{y}\left\|y-y^{\prime}\right\| .
$$

The latter readily implies the relationships

$$
\left\|x^{\prime}-\bar{x}\right\| \leq\left\|x-x^{\prime}\right\|+\|x-\bar{x}\| \leq 2 a \kappa \eta_{y}+\kappa\left(\eta_{y}+1\right) a=\left(3 \eta_{y}+1\right) \kappa a \leq \alpha,
$$

and therefore $y \in S(x) \cap \mathbb{B}_{\alpha}(\bar{y})=s(x)$ and $y^{\prime} \in S\left(x^{\prime}\right) \cap \mathbb{B}_{\alpha}(\bar{y})=s\left(x^{\prime}\right)$. Now we get from (4.5) that

$$
\left\|y-y^{\prime}\right\|=\left\|s(x)-s\left(x^{\prime}\right)\right\| \leq \ell\left\|x-x^{\prime}\right\| \leq \ell \kappa \eta_{y}\left\|y-y^{\prime}\right\| .
$$

It yields, since $\ell \kappa \eta_{y}<1$, that $y=y^{\prime}$ and thus completes the proof of assertion (i).

In order to prove assertion (ii), suppose that $Q$ is strongly regular around $(\bar{y}, \bar{z})$. Take some constants $\kappa>\operatorname{lip} Q(\bar{y}, \bar{z}), \eta_{x}>\widehat{\operatorname{lip}}_{x} f(\bar{x}, \bar{y})$, and $\eta_{y}>\widehat{\operatorname{lip}}_{y} f(\bar{x}, \bar{y})$ with $\kappa \eta_{y}<1$. By Theorem 2.2 we know that $S$ is Lipschitz-like around $(\bar{x}, \bar{y})$ with the exact bound estimate (4.4). Hence it remains to prove that there is a single-valued localization of $S$ around $\bar{x}$ that is nowhere multivalued, being thus single-valued due to its Lipschitz-like property. 
To proceed, choose a constant $\alpha>0$ and a mapping $g: Z \rightarrow Y$ such that $g(z)=Q^{-1}(z) \cap \mathbb{B}_{\alpha}(\bar{y})$ for $z \in \mathbb{B}_{\alpha}(\bar{z})$ with the estimates

$$
\begin{gathered}
\left\|g(z)-g\left(z^{\prime}\right)\right\| \leq \kappa\left\|z-z^{\prime}\right\| \text { for all } z, z^{\prime} \in \mathbb{B}_{\alpha}(\bar{z}) \text { and } \\
\left\|f(x, y)-f\left(x^{\prime}, y^{\prime}\right)\right\| \leq \eta_{x}\left\|x-x^{\prime}\right\|+\eta_{y}\left\|y-y^{\prime}\right\| \text { for all }(x, y),\left(x^{\prime}, y^{\prime}\right) \in \mathbb{B}_{\alpha}(\bar{x}) \times \mathbb{B}_{\alpha}(\bar{y})
\end{gathered}
$$

Take further a positive constant $a \leq \alpha$ satisfying $\left(\eta_{x}+\eta_{y}\right) a \leq \alpha$ and suppose that there are $y, y^{\prime} \in S(x) \cap \mathbb{B}_{a}(\bar{y})$ for some $x \in \mathbb{B}_{a}(\bar{x})$. Then we get

$$
z:=-f(x, y) \in Q(y) \text { and } z^{\prime}:=-f\left(x, y^{\prime}\right) \in Q\left(y^{\prime}\right) .
$$

It follows from the estimates

$$
\|z-\bar{z}\|=\|f(x, y)-f(\bar{x}, \bar{y})\| \leq \eta_{x}\|x-\bar{x}\|+\eta_{y}\|y-\bar{y}\| \leq \alpha
$$

that $y \in Q^{-1}(z) \cap \mathbb{B}_{\alpha}(\bar{y})=g(z)$ and similarly $y^{\prime}=g\left(z^{\prime}\right)$. It holds furthermore that

$$
\left\|y-y^{\prime}\right\|=\left\|g(z)-g\left(z^{\prime}\right)\right\| \leq \kappa\left\|z-z^{\prime}\right\|=\kappa\left\|f(x, y)-f\left(x, y^{\prime}\right)\right\| \leq \kappa \eta_{y}\left\|y-y^{\prime}\right\| .
$$

Since $\kappa \eta_{y}<1$, we conclude that $y=y^{\prime}$ and thus complete the proof of the theorem.

As a direct consequence of Theorem 4.1 we get the following result concerning the preservation of strong metric regularity under Lipschitzian perturbations, i.e., a localized single-valued version of Theorem 2.3. A proof based on the contracting mapping principle can be found in [2, Theorem 5F.1].

Corollary 4.2 (strong regularity under Lipschitzian perturbations). Let $F: X \Rightarrow Y$ be a set-valued mapping between Banach spaces with locally closed graph around $(\bar{x}, \bar{y}) \in \operatorname{gph} F$, and let $F$ be strongly metrically regular around $(\bar{x}, \bar{y})$ with constant $\kappa>0$. Consider a mapping $g: X \rightarrow Y$ Lipschitz continuous around $\bar{x}$ with constant $\lambda \geq 0$ such that $\lambda<\kappa^{-1}$. Then the mapping $F+g$ is strongly metrically regular around $(\bar{x}, \bar{y}+g(\bar{x}))$ with constant $\kappa /(1-\kappa \lambda)$.

Proof. Apply Theorem 4.1 with $f(x, y)=-x+g(y)$ and $Q=F$.

A simple example presented in [1, Remark 5.5(ii)] illustrates that the metric subregularity of field mappings $Q$ in (1.1) does not generally imply the calmness property of solution maps $S$ in (1.2). Let us now show (Proposition 4.3) that such an implication holds in the case of strong metric subregularity of $Q$ and isolated calmness of $S$ in the general framework of (1.1). This gives an appropriate one-point counterpart of Theorem 4.1(ii) above.

Recall that a set-valued mapping $F: X \rightrightarrows Y$ has the isolated calmness property at $(\bar{x}, \bar{y})$ with constant $\ell \geq 0$ if there are neighborhoods $U$ of $\bar{x}$ and $V$ of $\bar{y}$ such that

$$
F(x) \cap V \subset \bar{y}+\ell\|x-\bar{x}\| \mathbb{B} \text { for all } x \in U .
$$

We have the following important relationship between the isolated calmness of solution maps and strong subregularity of fields in the framework of generalized equations (1.1).

Theorem 4.3 (isolated calmness of solution maps from strong subregularity of fields in generalized equations). Let the base mapping $f: X \times Y \rightarrow Z$ in (1.1) be calm at $(\bar{x}, \bar{y})$, and let the field mapping $Q: Y \rightrightarrows Z$ be strongly metrically subregular at $(\bar{y}, \bar{z})$ with $\bar{z}:=-f(\bar{x}, \bar{y}) \in Q(\bar{y})$. Assume in addition the fulfillment of the condition

$$
\operatorname{clm}_{y} f(\bar{x}, \bar{y}) \cdot \operatorname{subreg} Q(\bar{y}, \bar{z})<1 \text {. }
$$


Then the solution map $S$ has the isolated calmness property at $(\bar{x}, \bar{y})$ with the exact bound estimate

$$
\operatorname{clm} S(\bar{x}, \bar{y}) \leq \frac{\operatorname{subreg} Q(\bar{y}, \bar{z}) \cdot \widehat{\operatorname{cm}}_{x} f(\bar{x}, \bar{y})}{1-\operatorname{subreg} Q(\bar{y}, \bar{z}) \cdot \operatorname{clm}_{y} f(\bar{x}, \bar{y})}
$$

Proof. Take any $\kappa>\operatorname{subreg} Q(\bar{y}, \bar{z}), \eta_{x}>\widehat{\operatorname{clm}}_{x} f(\bar{x}, \bar{y})$, and $\eta_{y}>\operatorname{clm}_{y} f(\bar{x}, \bar{y})$ with $\kappa \eta_{y}<1$ by (4.8). Choose further some positive constant $a$ so that

$$
\begin{gathered}
\|y-\bar{y}\| \leq \kappa d(\bar{z}, Q(y)) \text { for all } y \in \mathbb{B}_{a}(\bar{y}) \text { and } \\
\|f(x, y)-f(\bar{x}, \bar{y})\| \leq \eta_{x}\|x-\bar{x}\|+\eta_{y}\|y-\bar{y}\| \text { for all }(x, y) \in \mathbb{B}_{a}(\bar{x}) \times \mathbb{B}_{a}(\bar{y})
\end{gathered}
$$

Picking then $x \in \mathbb{B}_{a}(\bar{x})$ and $y \in S(x) \cap \mathbb{B}_{a}(\bar{y})$, we get the inequalities

$$
\|y-\bar{y}\| \leq \kappa d(\bar{z}, Q(y)) \leq \kappa\|f(x, y)-f(\bar{x}, \bar{y})\| \leq \kappa\left(\eta_{x}\|x-\bar{x}\|+\eta_{y}\|y-\bar{y}\|\right),
$$

which imply in turn that

$$
\|y-\bar{y}\| \leq \frac{\kappa \eta_{x}}{1-\kappa \eta_{y}}\|x-\bar{x}\|
$$

By the arbitrary choice of the constants $\left(\kappa, \eta_{x}, \eta_{y}\right)$ as above, we arrive at the upper estimate (4.9) and thus complete the proof of the theorem.

Similarly to Definition 3.1 we say that a set-valued mapping $F: X \times Y \Rightarrow Z$ is strongly metrically regular with respect to $x$ uniformly in $y$ around $((\bar{x}, \bar{y}), \bar{z}) \in \operatorname{gph} F$ with constant $\kappa>0$ if there are neighborhoods $U$ of $\bar{x}, V$ of $\bar{y}$, and $W$ of $\bar{z}$ such that estimate (3.1) hold and the mapping $F^{-1}(\cdot, y)(z) \cap U$ is not multivalued for all $y \in V$ and $z \in W$.

The next proposition establishes a strong partial metric regularity counterpart of Theorem 3.8.

Proposition 4.4 (strong partial metric regularity of base mappings from Lipschitzian properties of implicit multifunctions). In addition to the assumptions of Theorem 3.8, suppose that the implicit multifunction $\Gamma$ in (2.7) admits a single-valued Lipschitzian localization around $(\bar{y}, \bar{w})$. Then $f$ is strongly metrically regular with respect to $x$ uniformly in $y$ around $(\bar{x}, \bar{y})$.

Proof. Without loss of generality, assume that the set $\Gamma(y, w) \cap \mathbb{B}_{\alpha}(\bar{x})$ is a singleton for every $y \in \mathbb{B}_{\alpha}(\bar{y})$ and $w \in \mathbb{B}_{\alpha}(\bar{w})$, for $\alpha>0$ chosen as in the proof of Theorem 3.8. Fix $y \in \mathbb{B}_{a}(\bar{y})$ and $z \in \mathbb{B}_{a}(f(\bar{x}, \bar{y}))$ and pick any $x, x^{\prime} \in f^{-1}(\cdot, y)(z) \cap \mathbb{B}_{a}(\bar{x})$, with $0<a \leq \alpha$ verifying (3.10). Following now the proof of Theorem 3.8, we find $w \in g^{-1}(-f(x, y)) \cap \mathbb{B}_{\alpha}(\bar{w})$. This gives $x, x^{\prime} \in \Gamma(y, w)$ due to $f(x, y)=z=f\left(x^{\prime}, y\right)$. The latter implies in turn that $x=x^{\prime}$ by the local single-valuedness of $\Gamma$ and thus completes the proof of the proposition.

Now we complement Proposition 3.2 with a natural condition ensuring the strong partial metric regularity of nonsmooth single-valued mappings.

Proposition 4.5 (sufficient conditions for strong partial metric regularity). In addition to the assumptions of Proposition 3.2, suppose that $A$ is invertible. Then $f$ is strongly metrically regular with respect to $x$ uniformly in $y$ around $(\bar{x}, \bar{y})$. 
Proof. Take $\beta>0$ from the proof of Proposition 3.2, then pick $y \in \mathbb{B}_{\beta}(\bar{y})$ and $x, x^{\prime} \in \mathbb{B}_{\beta}(\bar{x})$ such that $f(x, y)=f\left(x^{\prime}, y\right)$. Since $A$ is invertible, we have the equalities

$$
x=-A^{-1}\left(f(x, y)-f\left(x^{\prime}, y\right)-A x\right) \quad \text { and } \quad x^{\prime}=A^{-1}\left(A x^{\prime}\right),
$$

which yield the relationships

$$
\begin{aligned}
\left\|x-x^{\prime}\right\| & =\left\|-A^{-1}\left(f(x, y)-f\left(x^{\prime}, y\right)-A\left(x-x^{\prime}\right)\right)\right\| \\
& \leq\left\|A^{-1}\right\| \cdot\left\|f(x, y)-f\left(x^{\prime}, y\right)-A\left(x-x^{\prime}\right)\right\| \\
& \leq \mu \cdot \operatorname{reg} A\left\|x-x^{\prime}\right\| \leq \mu \gamma\left\|x-x^{\prime}\right\|,
\end{aligned}
$$

This implies in turn that $x=x^{\prime}$ by $\mu \gamma<1$. Hence the mapping $f^{-1}(\cdot, y)(z) \cap \mathbb{B}_{\beta}(\bar{x})$ is nowhere multivalued for every $y \in \mathbb{B}_{\beta}(\bar{y})$ and $z \in Z$. Then we are done due to Proposition 3.2.

When $f$ is strictly differentiable with respect to $x$ uniformly in $y$ at the reference point, we have the following characterization of strong partial metric regularity.

Corollary 4.6 (characterization of strong partial metric regularity of partially smooth mappings). Let $f: X \times Y \rightarrow Z$ be a mapping between Banach spaces, and let $(\bar{x}, \bar{y}) \in X \times Y$ be such that $f$ is continuous at $(\bar{x}, \bar{y})$ and strictly partially differentiable at this point with respect to $x$ uniformly in $y$. Then $f$ is strongly metrically regular with respect to $x$ uniformly in $y$ around $(\bar{x}, \bar{y})$ if and only if $\nabla_{x} f(\bar{x}, \bar{y})$ is invertible. In this case we have the relationships

$$
\operatorname{reg}_{x} f(\bar{x}, \bar{y})=\operatorname{reg} \nabla_{x} f(\bar{x}, \bar{y})=\left\|\left(\nabla_{x} f(\bar{x}, \bar{y})\right)^{-1}\right\| .
$$

Proof. To justify the "only if" part, we follow the arguments of Proposition 3.4 using now Corollary 4.2 instead of Theorem 2.3. The converse is an immediate consequence of Proposition 4.5. $\triangle$

The next proposition complements Theorem 3.5 providing an additional condition that ensures that the Lipschitzian implicit (multi)function (2.7) is in fact locally single-valued.

Proposition 4.7 (Lipschitzian implicit functions). Suppose in addition to the assumptions of Theorem 3.5 that the base mapping $f$ is strongly metrically regular with respect to $x$ uniformly in $y$ around $(\bar{x}, \bar{y})$. Then $\Gamma$ in (2.7) admits a Lipschitz continuous single-valued localization around $(\bar{y}, \bar{w})$ with the exact bound estimate

$$
\operatorname{lip} \Gamma(\bar{y}, \bar{w}) \leq \operatorname{reg}_{x} f(\bar{x}, \bar{y}) \cdot \max \left\{\widehat{\operatorname{lip}}_{y} f(\bar{x}, \bar{y}), \operatorname{lip} g(\bar{w})\right\} .
$$

That is, the inverse mapping $\Gamma^{-1}$ is strongly metrically regular around $(\bar{x},(\bar{y}, \bar{w}))$.

Proof. Observe that if there is some positive constant $a$ such that the mapping $f^{-1}(\cdot, y)(z) \cap \mathbb{B}_{a}(\bar{x})$ is not multivalued whenever $y \in \mathbb{B}_{a}(\bar{y})$ and $z \in \mathbb{B}_{a}(\bar{z})$, then the implicit multifunction $\Gamma$ must admit a nowhere multivalued graphical localization. The rest follows from Theorem 3.5.

The following consequence of Theorem 3.8 and Proposition 4.7 characterizes the local singlevaluedness of Lipschitzian multifunctions in (2.7).

Corollary 4.8 (characterizing single-valued Lipschitzian localization of implicit multifunctions). Let $f: X \times Y \rightarrow Z$ be a mapping between Banach spaces, let $(\bar{x}, \bar{y}) \in X \times Y$ be such that $f$ is locally Lipschitzian with respect to $y$ uniformly in $x$ on some neighborhood $U \times V$ of $(\bar{x}, \bar{y})$, 
and let $f(\cdot, y)$ be continuous on $U$ for each $y \in V$. Given a mapping $g: W \rightarrow Z$ between Banach spaces with $g(\bar{w})=-f(\bar{x}, \bar{y})$ for some $\bar{w} \in W$, suppose that $C(g(\bar{w}))<\infty$ for the relative condition number (3.11), i.e., $g$ is both Lipschitz continuous and metrically regular around $\bar{w}$. Then the setvalued mapping $\Gamma: Y \times W \Rightarrow X$ defined by (2.7) admits a Lipschitzian single-valued localization around $(\bar{y}, \bar{w})$ if and only if $f$ is strongly metrically regular with respect to $x$ uniformly in $y$. In this case we have the exact bound estimates (4.11) and

$$
\operatorname{reg}_{x} f(\bar{x}, \bar{y}) \leq \widehat{\operatorname{lip}}_{w} \Gamma(\bar{y}, \bar{w}) \cdot \operatorname{reg} g(\bar{w})
$$

Proof. Follows directly from Theorem 3.8 and Proposition 4.7.

Finally in this section, we establish two-sided relationships between (conversely to Theorem 4.1) strong metric regularity of solution maps and Lipschitzian single-valued localizations of field mappings in the framework of generalized equations (1.1).

Theorem 4.9 (strong regularity of solution maps via single-valued Lipschitzian localization of fields in generalized equations). Let $f: X \times Y \rightarrow Z$ be a mapping between Banach spaces, and let $(\bar{x}, \bar{y}) \in X \times Y$ be such that $f$ is Lipschitz continuous around this point. Consider a set-valued field mapping $Q: Y \rightrightarrows Z$ with $\bar{z}:=-f(\bar{x}, \bar{y}) \in Q(\bar{y})$. Then the following assertions are satisfied:

(i) If $f$ is metrically regular with respect to $x$ uniformly in $y$ around $(\bar{x}, \bar{y})$ and if the solution map $S$ in (1.2) is strongly metrically regular around $(\bar{x}, \bar{y})$, then the field mapping $Q$ in (1.1) has a Lipschitzian single-valued localization around $(\bar{y}, \bar{z})$ with the exact bound estimate

$$
\operatorname{lip} Q(\bar{y}, \bar{z}) \leq \widehat{\operatorname{lip}}_{x} f(\bar{x}, \bar{y}) \cdot \operatorname{reg} S(\bar{x}, \bar{y})+\widehat{\operatorname{lip}}_{y} f(\bar{x}, \bar{y}) .
$$

(ii) The converse implication holds when $f$ is strongly regular with respect to $x$ uniformly in $y$ around $(\bar{x}, \bar{y})$ : if $Q$ has a Lipschitzian single-valued localization around $(\bar{y}, \bar{z})$, then $S$ is strongly metrically regular around $(\bar{x}, \bar{y})$ with the exact bound estimate

$$
\operatorname{reg} S(\bar{x}, \bar{y}) \leq \operatorname{reg}_{x} f(\bar{x}, \bar{y}) \cdot\left[\operatorname{lip} Q(\bar{y}, \bar{z})+\widehat{\operatorname{lip}}_{y} f(\bar{x}, \bar{y})\right]
$$

Proof. Observe first that the assumptions made in the theorem ensure the fulfillment of all the requirements of Theorem 3.5 with $W=Z$ and $g(z)=z$. Thus for any $\eta_{y}>\widehat{\operatorname{lip}}_{y} f(\bar{x}, \bar{y})$ and $\kappa>\widehat{\operatorname{reg}}_{x} f(\bar{x}, \bar{y})$ there is a positive constant $\alpha$ such that

$$
\Gamma\left(y^{\prime}, z^{\prime}\right) \cap \mathbb{B}_{\alpha}(\bar{x}) \subset \Gamma(y, z)+\kappa\left(\eta_{y}\left\|y-y^{\prime}\right\|+\left\|z-z^{\prime}\right\|\right) \mathbb{B}
$$

whenever $(y, z),\left(y^{\prime}, z^{\prime}\right) \in \mathbb{B}_{\alpha}(\bar{y}) \times \mathbb{B}_{\alpha}(\bar{z})$. To justify assertion (i), suppose that the solution map $S$ is strongly regular around $(\bar{x}, \bar{y})$ with a positive constant $\kappa$ and neighborhoods $U=\mathbb{B}_{a}(\bar{x})$ and $V=\mathbb{B}_{a}(\bar{y})$ for some $0<a \leq \alpha$. Due to Theorem 3.6(i) it is sufficient to prove the existence of a positive constant $b$ such that the mapping $y \mapsto Q(y) \cap \mathbb{B}_{b}(\bar{z})$ is not multivalued for any $y \in \mathbb{B}_{b}(\bar{y})$. To proceed, select $b>0$ such that

$$
\kappa\left(\eta_{y}+1\right) b \leq a
$$

and suppose that $z, z^{\prime} \in Q(y) \cap \mathbb{B}_{b}(\bar{z})$ for some $y \in \mathbb{B}_{b}(\bar{y})$. By (4.15) we find $x \in \Gamma(y, z)$ satisfying

$$
\|x-\bar{x}\| \leq \kappa\left(\eta_{y}\|y-\bar{y}\|+\|z-\bar{z}\|\right) \leq a,
$$


and hence $x \in S^{-1}(y) \cap \mathbb{B}_{a}(\bar{x})$. Employing further the same arguments gives us $x^{\prime} \in S^{-1}(y) \cap \mathbb{B}_{a}(\bar{x})$. This ensures that $x=x^{\prime}$ due to the single-valuedness property entailed by the strong regularity of $S$ and therefore justifies assertion (i).

To prove (ii), take $\eta_{x}>\widehat{\operatorname{lip}}_{x} f(\bar{x}, \bar{y})$ and suppose that $y \mapsto Q(y) \cap \mathbb{B}_{a}(\bar{z})$ is not multivalued for any $y \in \mathbb{B}_{a}(\bar{y})$, where $a$ is a positive constant with

$$
\left\|f(x, y)-f\left(x^{\prime}, y^{\prime}\right)\right\| \leq \eta_{x}\left\|x-x^{\prime}\right\|+\eta_{y}\left\|y-y^{\prime}\right\| \text { for all }(x, y),\left(x^{\prime}, y^{\prime}\right) \in \mathbb{B}_{a}(\bar{x}) \times \mathbb{B}_{a}(\bar{y}) .
$$

Make $a>0$ smaller if necessary so that the mapping $f^{-1}(\cdot, y)(z) \cap \mathbb{B}_{a}(\bar{x})$ is not multivalued for every $(y, z) \in \mathbb{B}_{a}(\bar{y}) \times \mathbb{B}_{a}(-\bar{z})$. Take further a positive constant $b \leq a$ such that $\left(\eta_{x}+\eta_{y}\right) b \leq a$ and let $x, x^{\prime} \in S^{-1}(y) \cap \mathbb{B}_{b}(\bar{x})$ for some $y \in \mathbb{B}_{b}(\bar{y})$. Then we get the inequalities

$$
\|-f(x, y)-\bar{z}\| \leq \eta_{x}\|x-\bar{x}\|+\eta_{y}\|y-\bar{y}\| \leq\left(\eta_{x}+\eta_{y}\right) b \leq a .
$$

The latter gives $-f(x, y) \in Q(y) \cap \mathbb{B}_{a}(\bar{z})$. Similarly we obtain $-f\left(x^{\prime}, y\right) \in Q(y) \cap \mathbb{B}_{a}(\bar{z})$ having hence $z:=f(x, y)=f\left(x^{\prime}, y\right)$. Since $x, x^{\prime} \in f^{-1}(\cdot, y)(z) \cap \mathbb{B}_{a}(\bar{x})$ and $(y, z) \in \mathbb{B}_{a}(\bar{y}) \times \mathbb{B}_{a}(-\bar{z})$, it follows that $x=x^{\prime}$. Applying now Theorem 3.6(i), we complete the proof of this theorem.

Remark 4.10 (relationships between strong regularity of base and solution maps in generalized equations). It is important to observe that the strong regularity assumption (or invertibility of $\nabla_{x} f(\bar{x}, \bar{y})$ when $f$ is strictly differentiable at $(\bar{x}, \bar{y})$ with respect to $\left.x\right)$ is not a superfluous condition. To illustrate this, consider a function $f: \mathbb{R}^{2} \times \mathbb{R} \rightarrow \mathbb{R}$ and a mapping $Q: \mathbb{R} \rightrightarrows \mathbb{R}$ defined by

$$
f\left(\left(x_{1}, x_{2}\right), y\right):=\alpha\left(x_{1}+x_{2}+y\right) \text { as } \alpha>0 \text { and } Q: \equiv 0 .
$$

Then $f$ is smooth everywhere with the surjective (but not invertible) partial derivative with respect to $x=\left(x_{1}, x_{2}\right)$. Also this function is Lipschitz continuous with constant $\alpha$, which can be chosen arbitrarily small. We can see furthermore that the mapping $Q$ is Lipschitzian with modulus 0 , while the solution map $S\left(x_{1}, x_{2}\right)=-x_{1}-x_{2}$ is not strongly regular around the origin.

\section{$5 \quad$ Metric hemiregularity and strong hemiregularity}

In this concluding section we define and study another useful version of metric regularity, where the domain point $\bar{x}$ is fixed in (2.1) instead of the range point $\bar{y}$ as in the case of subregularity (2.2). The new property and its subsequent partial and strong counterparts are important for a number of well-posedness issues in variational analysis and optimization, particularly for quantitative stability of solution maps to the parametric variational systems considered in what follows.

Definition 5.1 (metric hemiregularity of set-valued mappings). Given a set-valued mapping $F: X \rightrightarrows Y$ between Banach spaces and a point $(\bar{x}, \bar{y}) \in \operatorname{gph} F$, we say that $F$ is METRICALLY HEMIREGULAR at $(\bar{x}, \bar{y})$ with constant $\kappa>0$ if there is a neighborhood $V \subset Y$ of $\bar{y}$ such that

$$
d\left(\bar{x}, F^{-1}(y)\right) \leq \kappa\|y-\bar{y}\| \text { for all } y \in V \text {. }
$$

The infimum of $\kappa>0$ over all the combinations $(\kappa, U, V)$ for which (5.1) holds is called the EXACT HEMIREGULARITY BOUND of $F$ at $(\bar{x}, \bar{y})$ and is denoted hemreg $F(\bar{x}, \bar{y})$. 
Estimate (5.1) was mentioned in [3, p. 10] as the "Lipschitz lower semicontinuity" of the inverse mapping while, to the best of our knowledge, it has not been much studied and/or applied. We can easily see that the metric hemiregularity of $F$ yields the inner/lower semicontinuity of the inverse mapping $F^{-1}$ : for every neighborhood $U$ of $\bar{x}$ there is a neighborhood $V$ of $\bar{y}$ such that

$$
F^{-1}(y) \cap U \neq \emptyset \text { for all } y \in V .
$$

It follows immediately from the definitions that the metric regularity of $F$ around $(\bar{x}, \bar{y})$ always implies the metric hemiregularity of $F$ at this point, but not vice versa. We show now that for linear bounded operators both notions agree, with the same exact (hemi)regularity bound.

Proposition 5.2 (hemiregularity of linear bounded operators). A linear bounded operator $A \in \mathcal{L}(X, Y)$ is metrically hemiregular at every point $\bar{x} \in X$ if and only if it is surjective. In this case we have the relationships

$$
\text { hemreg } A=\operatorname{reg} A=\left\|\left(A^{*}\right)^{-1}\right\|,
$$

where hemreg $A$ stands for the common exact hemiregularity bound of $A$ at all the points $\bar{x} \in X$.

Proof. Observe first the obvious lower estimate

$$
\text { hemreg } A(\bar{x}) \leq \operatorname{reg} A \text { for every point } \bar{x} \in X \text {. }
$$

On the other hand, for any $\kappa>$ hemreg $A(\bar{x})$ there is some $a>0$ such that

$$
d\left(\bar{x}, A^{-1}(y)\right) \leq \kappa\|y-\bar{y}\| \text { for all } y \in \mathbb{B}_{a}(\bar{y})
$$

with $\bar{y}:=A \bar{x}$. Then we have that $w:=a y+\bar{y} \in \mathbb{B}_{a}(\bar{y})$ for all $y \in \mathbb{B}$, and hence

$$
a d\left(0, A^{-1}(y)\right)=d\left(\bar{x}, A^{-1}(a y+A \bar{x})\right)=d\left(\bar{x}, A^{-1}(w)\right) \leq \kappa\|w-\bar{y}\|=\kappa a .
$$

The latter implies in turn that

$$
\operatorname{reg} A=\sup _{y \in \mathbb{B}} d\left(0, A^{-1}(y)\right) \leq \kappa .
$$

Since $\kappa>$ hemreg $A(\bar{x})$ was chosen arbitrarily, we get the upper estimate hemreg $A(\bar{x}) \geq \operatorname{reg} A$ and thus justify the first equality in (5.2). The second one and the surjectivity characterization of metric regularity are well known; cf. the proof of Proposition 3.4.

Consider now a partial version of metric hemiregularity for mappings of two variables.

Definition 5.3 (partial metric hemiregularity). A set-valued mapping $F: X \times Y \Rightarrow Z$ is METRICALLY HEMIREGULAR WITH RESPECT TO $x$ UNIFORMLY IN $y$ at $((\bar{x}, \bar{y}), \bar{z}) \in \operatorname{gph} F$ with constant $\kappa>0$ if there are neighborhoods $V$ of $\bar{y}$ and $W$ of $\bar{z}$ such that

$$
d\left(\bar{x}, F^{-1}(\cdot, y)(z)\right) \leq \kappa d(z, F(\bar{x}, y)) \text { for all } y \in V \text { and } z \in W .
$$

The infimum of $\kappa>0$ over all the combinations $(\kappa, V, W)$ for which (5.3) holds is called the EXACT PARTIAL UNIFORM HEMIREGULARITY BOUND of $F$ in $x$ at $(\bar{x}, \bar{y})$ and is denoted hemreg ${ }_{x} F((\bar{x}, \bar{y}), \bar{z})$. 
Let us show that the property of (partial) hemiregularity for base mappings of the parametric generalized equations (1.1) is helpful to establish the converse assertion to Theorem 4.3. First we present a hemiregularity counterpart of Theorem 3.5 on implicit multifunctions, which is certainly of its independent interest.

Theorem 5.4 (implicit multifunctions under hemiregularity.) Let $f: X \times Y \rightarrow Z$ be a mapping between Banach spaces, and let $(\bar{x}, \bar{y}) \in X \times Y$ be such that $f(\cdot, y)$ is continuous on $U$ for each $y \in V$ for some neighborhoods $U$ of $\bar{x}$ and $V$ of $\bar{y}$. Given a mapping $g: W \rightarrow Z$ between Banach spaces with $g(\bar{w})=-f(\bar{x}, \bar{y})$ for some $\bar{w} \in W$, consider the implicit multifunction mapping $\Gamma: Y \times W \Rightarrow X$ defined in (2.7). Assume further that $f$ is metrically hemiregular with respect to $x$ uniformly in $y$ at $(\bar{x}, \bar{y})$ with constant $\kappa>0$, that $f$ is locally calm with respect to $y$ with constant $\eta \geq 0$ around $(\bar{x}, \bar{y})$, and that $g$ is locally calm around $\bar{w} \in W$ with constant $\lambda$. Then there is $\alpha>0$ such that for every $(y, w) \in \mathbb{B}_{\alpha}(\bar{y}) \times \mathbb{B}_{\alpha}(\bar{w})$ there exists $x \in \Gamma(y, w)$ satisfying

$$
\|x-\bar{x}\| \leq \kappa(\eta\|y-\bar{y}\|+\lambda\|w-\bar{w}\|) \text {. }
$$

The latter implies that $\Gamma^{-1}$ is metrically hemiregular at $(\bar{x},(\bar{y}, \bar{w}))$ with the following upper estimate of the exact hemiregularity bound:

$$
\text { hemreg } \Gamma^{-1}(\bar{x},(\bar{y}, \bar{w})) \leq \widehat{\operatorname{hemreg}}_{x} f(\bar{x}, \bar{y}) \cdot \max \left\{\operatorname{clm}_{y} f(\bar{x}, \bar{y}), \operatorname{clm} g(\bar{w})\right\} .
$$

Proof. Follows the one in Theorem 3.5 with $x^{\prime}=\bar{x}, y^{\prime}=\bar{y}$, and $w^{\prime}=\bar{w}$ therein. Note that in this setting only the calmness and hemiregularity assumptions are needed in comparison with the Lipschitz-like and regularity properties in Theorem 3.5.

Now we are ready to formulate and prove the aforementioned converse to Theorem 4.3.

Theorem 5.5 (strong subregularity of fields via isolated calmness of solution maps in generalized equations). Let $f: X \times Y \rightarrow Z$ be a base mapping of (1.1) in the arbitrary Banach space framework, let $(\bar{x}, \bar{y}) \in X \times Y$, and let $Q: Y \rightrightarrows Z$ be a set-valued field mapping with $\bar{z}:=-f(\bar{x}, \bar{y}) \in Q(\bar{y})$. Assume that $f$ is locally calm with respect to $y$ uniformly in $x$ on some neighborhood $U \times V$ of $(\bar{x}, \bar{y})$, that $f(\cdot, y)$ be continuous on $U$ for each $y \in V$, and that $f$ is metrically hemiregular with respect to $x$ uniformly in $y$ at $(\bar{x}, \bar{y})$. Then the field $Q$ is strongly metrically subregular at $(\bar{y}, \vec{z})$ provided that the solution map $S: X \Rightarrow Y$ in (1.2) has the isolated calmness property at $(\bar{x}, \bar{y})$ and that the condition

$$
\widehat{\operatorname{hemreg}}_{x} f(\bar{x}, \bar{y}) \cdot \operatorname{clm} S(\bar{x}, \bar{y}) \cdot \operatorname{clm}_{y} f(\bar{x}, \bar{y})<1
$$

is satisfied. In this case we have the exact bound estimate

$$
\text { subreg } Q(\bar{y}, \bar{z}) \leq \frac{\widehat{\operatorname{hemreg}}_{x} f(\bar{x}, \bar{y}) \cdot \operatorname{clm} S(\bar{x}, \bar{y})}{1-\widehat{\operatorname{hemreg}}_{x} f(\bar{x}, \bar{y}) \cdot \operatorname{clm} S(\bar{x}, \bar{y}) \cdot \operatorname{clm}_{y} f(\bar{x}, \bar{y})} .
$$

Proof. By (5.5), take $\ell>\operatorname{clm} S(\bar{x}, \bar{y}), \eta_{y}>\operatorname{clm}_{y} f(\bar{x}, \bar{y})$, and $\kappa>\widehat{\text { hemreg }_{x}} f(\bar{x}, \bar{y})$ with $\ell \kappa \eta_{y}<1$. Then choose a positive constant $a$ such that

$$
S(x) \cap \mathbb{B}_{a}(\bar{y}) \subset \bar{y}+\ell\|x-\bar{x}\| \mathbb{B} \text { for all } x \in \mathbb{B}_{a}(\bar{x}) .
$$

Consider the implicit multifunction

$$
\Gamma(y, z)=\{x \in X \mid f(x, y)+z=0\}
$$


and employ Theorem 5.4 to conclude that the inverse mapping $\Gamma^{-1}$ is metrically hemiregular at $(\bar{x},(\bar{y}, \bar{z}))$. Make $a>0$ smaller if necessary in order to ensure, for every $(y, z) \in \mathbb{B}_{a}(\bar{y}) \times \mathbb{B}_{a}(\bar{z})$, the existence of $x \in \Gamma(y, z)$ such that

$$
\|x-\bar{x}\| \leq \kappa\left(\eta_{y}\|y-\bar{y}\|+\|z-\bar{z}\|\right) .
$$

Pick further $y \in \mathbb{B}_{a}(\bar{y})$ and $z \in Q(y) \cap \mathbb{B}_{a}(\bar{z})$ observing that we are done if such $z$ does not exist. Then there is some $x \in \Gamma(y, z)$ satisfying (5.8). Hence $y \in S(x) \cap \mathbb{B}_{a}(\bar{y})$, and therefore

$$
\|y-\bar{y}\| \leq \ell\|x-\bar{x}\| \leq \ell \kappa\left(\eta_{y}\|y-\bar{y}\|+\|z-\bar{z}\|\right) .
$$

The latter implies the estimate

$$
\|y-\bar{y}\| \leq \frac{\ell \kappa}{1-\ell \kappa \eta_{y}}\|z-\bar{z}\| .
$$

Taking finally into account that the positive numbers $\ell, \eta_{y}$, and $\kappa$ can be chosen arbitrarily close to the exact bounds $\operatorname{clm} S(\bar{x}, \bar{y}), \operatorname{clm}_{y} f(\bar{x}, \bar{y})$, and $\widehat{\operatorname{hemreg}}_{x} f(\bar{x}, \bar{y})$, respectively, we conclude from (5.9) that the field $Q$ is strongly metrically subregular at $(\bar{y}, \bar{z})$ with the exact bound estimate (5.6). This completes the proof of the theorem.

Next we consider strong counterparts of the metric hemiregularity notion and its partial version.

Definition 5.6 (strong hemiregularity and partial strong hemiregularity). Given a setvalued mapping $F: X \rightrightarrows Y$ and a point $(\bar{x}, \bar{y}) \in \operatorname{gph} F$, we say that $F$ is STRONGLY METRICALLY HEMIREGULAR at $(\bar{x}, \bar{y})$ (or STRONGLY HEMIREGULAR at this point) with constant $\kappa>0$ if there are neighborhoods $U \subset X$ of $\bar{x}$ and $V \subset Y$ of $\bar{y}$ such that (5.1) holds and that $F^{-1}$ admits a single-valued localization on $U \times V$. Similarly to the above we define the (PARTIAL) STRONG HEMIREGULARITY property of $F$ with respect to $x$ uniformly in $y$ at $(\bar{x}, \bar{y})$ by replacing condition (3.1) by (5.3) in the definition of partial strong metric regularity.

It is easy to see that strong hemiregularity is weaker than strong regularity. Furthermore, we have the following equivalence relationships between the strong hemiregularity of the mapping in question and the calm single-valued localization of its inverse.

Proposition 5.7 (equivalence between strong hemiregularity of mappings and calm single-valued localization of their inverses). A mapping $F: X \Rightarrow Y$ is strongly hemiregular at some point $(\bar{x}, \bar{y}) \in \mathrm{gph} F$ if and only if $F^{-1}$ admits a calm single-valued localization $s(\cdot)$ at $(\bar{y}, \bar{x})$. Furthermore, we have the equality between the corresponding exact bounds

$$
\text { hemreg } F(\bar{x}, \bar{y})=\operatorname{clm} s(\bar{y}) \text {. }
$$

Proof. If $F$ is strongly hemiregular at $(\bar{x}, \bar{y}) \in \operatorname{gph} F$ with some constant $\kappa>$ hemreg $F(\bar{x}, \bar{y})$, then there is a positive number $a$ such that (5.1) holds and the set $F^{-1}(y) \cap \mathbb{B}_{a}(\bar{x})$ is a singleton for $y \in \mathbb{B}_{a}(\bar{y})$. Take a mapping $s: Y \rightarrow X$ with $s(y)=F^{-1}(y) \cap \mathbb{B}_{a}(\bar{x})$ for $y \in \mathbb{B}_{a}(\bar{x})$. Let $\varepsilon>0$ and $0<\alpha \leq a$ be selected so that $(\kappa+\varepsilon) \alpha \leq a$. For $y \in \mathbb{B}_{\alpha}(\bar{y})$, there is $x \in F^{-1}(y)$ satisfying

$$
\|x-\bar{x}\| \leq(\kappa+\varepsilon)\|y-\bar{y}\| \leq(\kappa+\varepsilon) \alpha \leq a,
$$


which gives $s(y)=x$. Since $s(\bar{y})=\bar{x}$, we have

$$
\|s(y)-s(\bar{y})\|=\|x-\bar{x}\| \leq(\kappa+\varepsilon)\|y-\bar{y}\|,
$$

which justifies the calmness of $s(\cdot)$ and the inequality " $\geq$ " in (5.10) by the arbitrary choice of $\varepsilon>0$.

Conversely, suppose that there are constants $a>0$ and $\kappa \geq 0$ such that $F^{-1}(y) \cap \mathbb{B}_{a}(\bar{x})=s(y)$ and the calmness relationship

$$
\|s(y)-s(\bar{y})\| \leq \kappa\|y-\bar{y}\| \text { whenever } y \in \mathbb{B}_{a}(\bar{y})
$$

holds. Then for all $y \in \mathbb{B}_{a}(\bar{y})$ we have the estimates

$$
d\left(\bar{x}, F^{-1}(y)\right) \leq d\left(\bar{x}, F^{-1}(y) \cap \mathbb{B}_{a}(\bar{x})\right)=\|s(\bar{y})-s(y)\| \leq \kappa\|y-\bar{y}\|,
$$

which imply the inequality " $\leq$ " in (5.10) and thus complete the proof of the proposition.

Now we can get the following strong counterpart of Theorem 5.4.

Proposition 5.8 (implicit multifunctions under strong hemiregularity). In addition to the assumptions of Theorem 3.5, suppose that $f$ is strongly hemiregular with respect to $x$ uniformly in $y$ at $(\bar{x}, \bar{y})$. Then the implicit multifunction $\Gamma$ in (2.7) admits a calm single-valued localization at $((\bar{y}, \bar{w}), \bar{x})$, that is, $\Gamma^{-1}$ is strongly hemiregular at $(\bar{x},(\bar{y}, \bar{w}))$ with the exact bound estimate

$$
\text { hemreg } \Gamma^{-1}(\bar{x},(\bar{y}, \bar{w})) \leq \widehat{\operatorname{hemreg}} f(\bar{x}, \bar{y}) \cdot \max \left\{\operatorname{clm}_{y} f(\bar{x}, \bar{y}), \operatorname{clm} g(\bar{w})\right\} .
$$

Proof. Follows from Theorem 5.4, Definition 5.6, and Proposition 5.7.

Finally in this section, we establish a "one-point" counterpart of Theorem 4.9, where the (strong) metric hemiregularity assumption on the base mapping in (1.1) places an essential role.

Theorem 5.9 (strong subregularity of solution maps via isolated calmness of fields in generalized equations). Let $f: X \times Y \rightarrow Z$ be a mapping between Banach spaces, and let $(\bar{x}, \bar{y}) \in X \times Y$ be such that $f$ is calm at this point. Consider a set-valued field mapping $Q: Y \rightrightarrows Z$ in (1.1) with $\bar{z}:=-f(\bar{x}, \bar{y}) \in Q(\bar{y})$. Then the following assertions are satisfied:

(i) Suppose that base mapping $f$ is metrically hemiregular with respect to $x$ uniformly in $y$ at $(\bar{x}, \bar{y})$ and that the solution map $S$ in $(1.2)$ is strongly subregular at $(\bar{x}, \bar{y})$. Then the field $Q$ has the isolated calmness property at $(\bar{y}, \bar{z})$ with the exact bound estimate

$$
\operatorname{clm} Q(\bar{y}, \bar{z}) \leq \widehat{\operatorname{clm}}_{x} f(\bar{x}, \bar{y}) \cdot \operatorname{subreg} S(\bar{x}, \bar{y})+\operatorname{clm}_{y} f(\bar{x}, \bar{y}) .
$$

(ii) Assume in addition that $f$ is strongly hemiregular with respect to $x$ uniformly in $y$ around $(\bar{x}, \bar{y})$. Then we have the converse assertion to (i)): if $Q$ has the isolated calmness property at $(\bar{y}, \bar{z})$, then $S$ is strongly subregular at $(\bar{x}, \bar{y})$ with the exact bound estimate

$$
\text { subreg } S(\bar{x}, \bar{y}) \leq \widehat{\operatorname{hemreg}}_{x} f(\bar{x}, \bar{y}) \cdot\left[\operatorname{clm} Q(\bar{y}, \bar{z})+\operatorname{clm}_{y} f(\bar{x}, \bar{y})\right] .
$$

Proof. To proceed, apply the hemiregularity implicit multifunction result of Theorem 5.4. In this way we consider the mapping $\Gamma$ defined in (5.7) and for any numbers $\eta_{y}>\operatorname{clm}_{y} f(\bar{x}, \bar{y})$ and $\kappa>\widehat{\operatorname{hemreg}}_{x} f(\bar{x}, \bar{y})$ find a positive constant $\alpha$ such that whenever $(y, z) \in \mathbb{B}_{\alpha}(\bar{y}) \times \mathbb{B}_{\alpha}(\bar{z})$ there is $x \in \Gamma(y, z)$ satisfying

$$
\|x-\bar{x}\| \leq \kappa\left(\eta_{y}\|y-\bar{y}\|+\|z-\bar{z}\|\right)
$$


To prove assertion (i) of the theorem, we get by the strong subregularity of the solution map $S$ at $(\bar{x}, \bar{y})$ some positive constants $\ell$ and $a$ for which

$$
\|x-\bar{x}\| \leq \ell d(\bar{y}, S(x)) \text { whenever } x \in \mathbb{B}_{a}(\bar{x}) .
$$

Take further $\eta_{x}>\widehat{\operatorname{clm}}_{x} f(\bar{x}, \bar{y})$ and make $a>0$ smaller if necessary to have

$$
\|f(x, y)-f(\bar{x}, \bar{y})\| \leq \eta_{x}\|x-\bar{x}\|+\eta_{y}\|y-\bar{y}\| \text { for all }(x, y) \in \mathbb{B}_{a}(\bar{x}) \times \mathbb{B}_{a}(\bar{y}) .
$$

Next decrease $\alpha>0$ if necessary to make sure that

$$
\alpha \leq a \text { and } \kappa\left(\eta_{y}+1\right) \alpha \leq a .
$$

Then pick $y \in \mathbb{B}_{\alpha}(\bar{y})$ and $z \in Q(y) \cap \mathbb{B}_{\alpha}(\bar{z})$ observing that we are done if no such $z$ exists. By (5.13) we get $x \in \Gamma(y, z)$ such that

$$
\|x-\bar{x}\| \leq \kappa\left(\eta_{y}\|y-\bar{y}\|+\|z-\bar{z}\|\right) \leq \kappa\left(\eta_{y}+1\right) \alpha \leq a .
$$

Hence $y \in S(x)$ by the choice of $y$ and $z$, which allows us to conclude from (5.14) and (5.15) that

$$
\begin{aligned}
\|z-\bar{z}\| & =\|f(x, y)-f(\bar{x}, \bar{y})\| \leq \eta_{x}\|x-\bar{x}\|+\eta_{y}\|y-\bar{y}\| \leq \ell \eta_{x} d(\bar{y}, S(x))+\eta_{y}\|y-\bar{y}\| \\
& \leq\left(\ell \eta_{x}+\eta_{y}\right)\|y-\bar{y}\| .
\end{aligned}
$$

Since the constants $\eta_{x}$ and $\eta_{y}$ above can be chosen arbitrarily close to $\widehat{\operatorname{clm}}_{x} f(\bar{x}, \bar{y})$ and $\operatorname{clm}_{y} f(\bar{x}, \bar{y})$, respectively, while $\ell$ is arbitrarily close to subreg $S(\bar{x}, \bar{y})$, we arrive at the corresponding exact bound estimate (5.11) and thus complete the proof of assertion (i) of the theorem.

To justify now the converse assertion (ii), suppose that $Q$ has the isolated calmness property at $(\bar{y}, \bar{z})$, i.e., we have the inclusion

$$
Q(y) \cap \mathbb{B}_{a}(\bar{z}) \subset \bar{z}+\ell\|y-\bar{y}\| \mathbb{B} \text { whenever } y \in \mathbb{B}_{a}(\bar{y})
$$

with some constants $\ell \geq 0$ and $a>0$. Pick any $\eta_{x}>\widehat{\operatorname{clm}}_{x} f(\bar{x}, \bar{y})$ and make $a$ smaller if necessary to ensure the estimate

$$
\|f(x, y)-f(\bar{x}, \bar{y})\| \leq \eta_{x}\|x-\bar{x}\|+\eta_{y}\|y-\bar{y}\| \text { for all }(x, y) \in \mathbb{B}_{a}(\bar{x}) \times \mathbb{B}_{a}(\bar{y}) .
$$

Taking into account Proposition 5.8 involving the strong hemiregularity property of the base mapping $f$, we choose $\alpha>0$ in (5.13) with $\alpha \leq a$ and such that the set $\Gamma(y, z) \cap \mathbb{B}_{\alpha}(\bar{x})$ is a singleton for every $(y, z) \in \mathbb{B}_{\alpha}(\bar{y}) \times \mathbb{B}_{\alpha}(\bar{z})$. Then select $\beta>0$ satisfying the inequalities

$$
\beta \leq \alpha, \quad\left(\eta_{x}+\eta_{y}\right) \beta \leq \alpha, \text { and }\left(\eta_{x}+2 \eta_{y}\right) \kappa \beta \leq \alpha .
$$

Fix further $x \in \mathbb{B}_{\beta}(\bar{x})$ and $y \in S(x) \cap \mathbb{B}_{\beta}(\bar{y})$ observing that there is nothing to prove if such a point $x$ does not exist. Then for $z:=-f(x, y)$ we have $z \in Q(y)$ and

$$
\|z-\bar{z}\|=\|f(x, y)-f(\bar{x}, \bar{y})\| \leq \eta_{x}\|x-\bar{x}\|+\eta_{y}\|y-\bar{y}\| \leq\left(\eta_{x}+\eta_{y}\right) \beta \leq \alpha .
$$

Thus it follows from (5.13) the existence of some $\widetilde{x} \in \Gamma(y, z)$ satisfying the estimates

$$
\|\tilde{x}-\bar{x}\| \leq \kappa\left(\eta_{y}\|y-\bar{y}\|+\|z-\bar{z}\|\right) \leq\left(\eta_{x}+2 \eta_{y}\right) \kappa \beta \leq \alpha .
$$

The latter give that $\widetilde{x} \in \Gamma(y, z) \cap \mathbb{B}_{\alpha}(\bar{x})=\{x\}$, i.e., $\widetilde{x}=x$. Finally, from (5.16) and (5.17) we get

$$
\|x-\bar{x}\| \leq \kappa\left(\eta_{y}\|y-\bar{y}\|+\|z-\bar{z}\|\right) \leq \kappa\left(\eta_{y}+\ell\right)\|y-\bar{y}\|,
$$

which implies by the arbitrary choice of $\kappa, \eta_{y}$, and $\ell$ as above that the solution map $S$ is strongly subregular at $(\bar{x}, \bar{y})$ with the exact bound estimate (5.12). This justifies assertion (ii) and completes the proof of the theorem. 
Remark 5.10 (relationships between strong hemiregularity of bases and strong subregularity of solution maps in generalized equations). It is worth to make the following observations concerning the assumptions and results obtained in Theorem 5.9.

(i) Note first the strong hemiregularity assumption on the base mapping $f$ is essential for the conclusion in (ii) of the theorem. Indeed, consider a function $f: \mathbb{R}^{2} \times \mathbb{R} \rightarrow \mathbb{R}$ as in Remark 4.10(i) and the field mapping $Q$ in (1.1) with $\operatorname{gph} Q=\{(0,0)\}$. Then $f$ is smooth, Lipschitzian while not strongly hemiregular at $(0,0)$. On the other hand, the field $Q$ has the isolated calmness property at $(0,0)$ with modulus 0 , but the corresponding solution map

$$
S\left(x_{1}, x_{2}\right)= \begin{cases}0 & \text { if } x_{1}=-x_{2} \\ \emptyset & \text { otherwise }\end{cases}
$$

is not strongly subregular at $((0,0), 0)$, since $0 \in S(\varepsilon,-\varepsilon)$ for all $\varepsilon>0$.

(ii) Observe that $S$ can be strongly subregular and $Q$ can have the isolated calmness property while $f$ may not be metrically hemiregular with respect to $x$ uniformly in $y$. This means that the converse implication like in Proposition 4.4 does not hold. The following example of (1.1) with $f: \mathbb{R} \times \mathbb{R}^{2} \rightarrow \mathbb{R}^{2}$ and $Q: \mathbb{R}^{2} \rightarrow \mathbb{R}^{2}$ given by

$$
f\left(x,\left(y_{1}, y_{2}\right)\right)=(-x,-x) \text { and } Q\left(y_{1}, y_{2}\right)=\left(y_{1}, y_{2}\right)
$$

illustrates it. Indeed, we have here that the solution map $S(x)=(x, x)$ is strongly subregular and the field $Q$ has the isolated calmness property around any point of their graph while

$$
f^{-1}\left(\cdot,\left(y_{1}, y_{2}\right)\right)\left(z_{1}, z_{2}\right)=\emptyset \text { for every } z_{1} \neq z_{2}
$$

Acknowledgements. Research of the first author was partially supported by MICINN of Spain, grant MTM2008-06695-C03-01 and program "Juan de la Cierva." Research of the second author was partially supported by the US National Science Foundation under grant DMS-0603846.

\section{References}

[1] Aragón Artacho, F.J., Mordukhovich, B.S.: Metric regularity and lipschitzian stability of parametric variational inequalities. Nonlinear Anal. 72, 1149-1170 (2010)

[2] Dontchev, A.L., Rockafellar, R.T.: Implicit Functions and Solution Mappings. Springer, Dordrecht (2009)

[3] Klatte, D., Kummer, B.: Nonsmooth Equations in Optimization: Regularity, Calculus and Applications. Kluwer, Boston (2002)

[4] Mordukhovich, B.S.: Variational Analysis and Generalized Differentiation, I: Basic Theory, II: Applications. Springer, Berlin (2006)

[5] Renegar, J.: Linear programming, complexity theory and elementary functional analysis. Math. Program. 70, 279-351 (1995)

[6] Robinson, S.M.: Strongly regular generalized equations. Math. Oper. Res. 5, 43-62 (1980) 